\title{
LOGIC PROGRAMMING
}

\author{
Robert Kowalski
}

\section{INTRODUCTION}

The driving force behind logic programming is the idea that a single formalism suffices for both logic and computation, and that logic subsumes computation. But logic, as this series of volumes proves, is a broad church, with many denominations and communities, coexisting in varying degrees of harmony. Computing is, similarly, made up of many competing approaches and divided into largely disjoint areas, such as programming, databases, and artificial intelligence.

On the surface, it might seem that both logic and computing suffer from a similar lack of cohesion. But logic is in better shape, with well-understood relationships between different formalisms. For example, first-order logic extends propositional logic, higher-order logic extends first-order logic, and modal logic extends classical logic. In contrast, in Computing, there is hardly any relationship between, for example, Turing machines as a model of computation and relational algebra as a model of database queries. Logic programming aims to remedy this deficiency and to unify different areas of computing by exploiting the greater generality of logic. It does so by building upon and extending one of the simplest, yet most powerful logics imaginable, namely the logic of Horn clauses.

In this paper, which extends a shorter history of logic programming (LP) in the 1970s [Kowalski, 2013], I present a personal view of the history of LP, focusing on logical, rather than on technological issues. I assume that the reader has some background in logic, but not necessarily in LP. As a consequence, this paper might also serve a secondary function, as a survey of some of the main developments in the logic of LP.

Inevitably, a history of this restricted length has to omit a number of important topics. In this case, the topics omitted include meta LP, high-order LP, concurrent LP, disjunctive LP and complexity. Other histories and surveys that cover some of these topics and give other perspectives include [Apt and Bol, 1994; Brewka et al., 2011; Bry et al. 2007; Ceri et al., 1990; Cohen, 1988; Colmerauer and Roussel, 1996; Costantini, 2002; Dantsin et al., 1997; Eiter et al., 2009; Elcock, 1990; van Emden, 2006; Hewitt, 2009; Minker, 1996; Ramakrishnan and Ullman, 1993].

Perhaps more significantly and more regrettably, in omitting coverage of technological issues, I may be giving a misleading impression of their significance. Without Colmerauer's practical insights [Colmerauer et al., 1973], Boyer and Moore's 
[1972] structure sharing implementation of resolution [Robinson, 1965a], and Warren's abstract machine and Prolog compiler [Warren, 1978, 1983; Warren et al., 1977], logic programming would have had far less impact in the field of Computing, and this history would not be worth writing.

\subsection{The Horn clause basis of logic programming}

Horn clauses are named after the logician Alfred Horn, who studied some of their mathematical properties. A Horn clause logic program is a set of sentences (or clauses) each of which can be written in the form:

$$
A_{0} \leftarrow A_{1} \wedge \ldots \wedge A_{n} \text { where } n \geq 0
$$

Each $A_{i}$ is an atomic formula of the form $p\left(t_{1}, \ldots, t_{m}\right)$, where $p$ is a predicate symbol and the $t_{i}$ are terms. Each term is either a constant symbol, variable, or composite term of the form $f\left(t_{1}, \ldots, t_{m}\right)$, where $f$ is a function symbol and the $t_{i}$ are terms. Every variable occurring in a clause is universally quantified, and its scope is the clause in which the variable occurs. The backward arrow $\leftarrow$ is read as "if", and $\wedge$ as "and". The atom $A_{0}$ is called the conclusion (or head) of the clause, and the conjunction $A_{1} \wedge \ldots \wedge A_{n}$ is the body of the clause. The atoms $A_{1}, \ldots, A_{n}$ in the body are called conditions. If $n=0$, then the body is equivalent to true, and the clause $A_{0} \leftarrow$ true is abbreviated to $A_{0}$ and is called a fact. Otherwise if $n \neq 0$, the clause is called a rule.

It is also useful to allow the head $A_{0}$ of a clause to be false, in which case, the clause is abbreviated to $\leftarrow A_{1} \wedge \ldots \wedge A_{n}$ and is called a goal clause. Intuitively, a goal clause can be understood as denying that the goal $A_{1} \wedge \ldots \wedge A_{n}$ has a solution, thereby issuing a challenge to refute the denial by finding a solution.

Predicate symbols represent the relations that are defined (or computed) by a program, and functions are treated as a special case of relations, as in relational databases. Thus the mother function, exemplified by mother(john) $=$ mary, is represented by a fact such as mother(john, mary). The definition of maternal grandmother, which in functional notion is written as an equation:

$$
\text { maternal-grandmother }(X)=\text { mother }(\operatorname{mother}(X))
$$

is written as a rule in relational notation:

$$
\text { maternal-grandmother }(X) \leftarrow \text { mother }(X, Z) \wedge \operatorname{mother}(Z, Y)^{1}
$$

Although all variables in a rule are universally quantified, it is often more natural to read variables in the conditions that are not in the conclusion as existentially quantified with the body of the rule as their scope. For example, the following two sentences are equivalent:

\footnotetext{
${ }^{1}$ In this paper, I use the Prolog notation for clauses: Predicate symbols, function symbols and constants start with a lower case letter, and variables start with an upper case letter. Numbers can be treated as constants.
} 
$\forall X Y Z[$ maternal-grandmother $(X) \leftarrow \quad$ mother $(X, Z) \wedge$ mother $(Z, Y)]$

$\forall X Y \quad[$ maternal-grandmother $(X) \leftarrow \exists Z[$ mother $(X, Z) \wedge$ mother $(Z, Y)]]$

Function symbols are not used for function definitions, but are used to construct composite data structures. For example, the composite term cons $(s, t)$ can be used to construct a list with first element $s$ followed by the list $t$. Thus the term cons (john, cons(mary, nil)) represents the list [john, mary], where nil represents the empty list.

Terms can contain variables, and logic programs can compute input-output relations containing variables. However, for the semantics, it is convenient to regard terms that do not contain variables, called ground terms, as the basic data structures of logic programs. Similarly, a clause or other expression is said to be ground, if it does not contain any variables.

Logic programs that do not contain function symbols are also called Datalog programs. Datalog is more expressive than relational databases, but is also decidable. Horn clause programs with function symbols have the expressive power of Turing machines, and consequently are undecidable. Horn clauses are sufficient

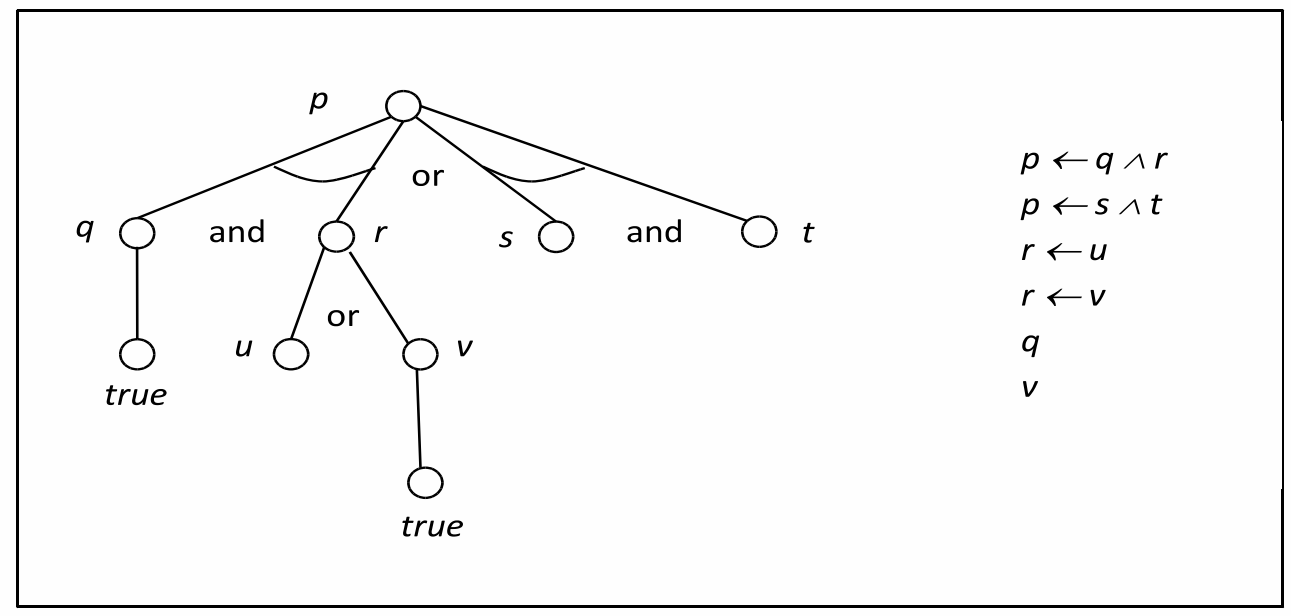

Figure 1. An and-or tree and corresponding propositional Horn clause program.

for many applications in artificial intelligence. For example, and-or trees can be represented by ground Horn clauses. ${ }^{2}$ See figure 1.

\footnotetext{
${ }^{2}$ And-or trees were employed in many early artificial intelligence programs, including the geometry theorem proving machine of Gelernter [1963]. Search strategies for and-or trees were investigated by Nils Nilsson [1968], and in a theorem-proving context by Kowalski [1970].
} 


\subsection{Logic programs with negation}

Although Horn clauses are the underlying basis of LP and are theoretically sufficient for all programming and database applications, they are not adequate for artificial intelligence, most importantly because they fail to capture non-monotonic reasoning. For non-monotonic reasoning, it is necessary to extend Horn clauses to clauses of the form:

$$
A_{0} \leftarrow A_{1} \wedge \ldots \wedge A_{n} \wedge \text { not } B_{1} \wedge \ldots \wedge \text { not } B_{m} \text { where } n \geq 0 \text { and } m \geq 0 .
$$

Each $A_{i}$ and $B_{i}$ is an atomic formula, and "not" is read as not. Atomic formulas and their negations are also called literals. Here the $A_{i}$ are positive literals, and the not $B_{i}$ are negative literals. Sets of clauses in this form are called normal logic programs, or just logic programs for short.

Normal logic programs, with appropriate semantics for negation, are sufficient to solve the frame problem in artificial intelligence. Here is a solution using an LP representation of the situation calculus [McCarthy and Hayes, 1969]:

$$
\begin{aligned}
& \operatorname{holds}(F, \operatorname{do}(A, S)) \leftarrow \operatorname{poss}(A, S) \wedge \operatorname{initiates}(A, F, S) \\
& \operatorname{holds}(F, d o(A, S)) \leftarrow \operatorname{poss}(A, S) \wedge \operatorname{holds}(F, S) \wedge \operatorname{not} \operatorname{terminates}(A, F, S)
\end{aligned}
$$

Here $\operatorname{holds}(F, S)$ expresses that a fact $F$ (also called a fluent) holds in a state (or situation) $S ; \operatorname{poss}(A, S)$ that the action $A$ is possible in state $S ;$ initiates $(A, F, S)$ that the action $A$ performed in state $S$ initiates $F$ in the resulting state $\operatorname{do}(A, S)$; and terminates $(A, F, S)$ that $A$ terminates $F$. Together, the two clauses assert that a fact holds in a state either if it is initiated by an action or if it held in the previous state and was not terminated by an action.

This representation of the situation calculus also illustrates meta-logic programming, because the predicates holds, poss, initiates and terminates can be understood as meta-predicates, where the variable $F$ ranges over names of sentences. Alternatively, they can be interpreted as second-order predicates, where $F$ ranges over first-order predicates.

\subsection{Logic programming issues}

In this article, I will discuss the development of LP and its extensions, their semantics, and their proof theories. We will see that lurking beneath the deceptively simple syntax of logic programs are profound issues concerning semantics, proof theory and knowledge representation.

For example, what does it mean for a logic program $P$ to solve a goal $G$ ? Does it mean that $P$ logically implies $G$, in the sense that $G$ is true in all models of $P$ ? Does it mean that some larger theory than $P$, which includes assumptions implicit in $P$, logically implies $G$ ? Or does it mean that $G$ is true in some natural, intended model of $P$ ?

And how should $G$ be solved? Top-down by using the clauses in $P$ as goalreduction procedures, to reduce goals that match the conclusions of clauses to 
sub-goals that correspond to their conditions? Or bottom-up to generate new conclusions from conditions, until the generated conclusions include all the information needed to solve the goal $G$ in one step?

We will see that these two issues - what it means to solve a goal $G$, and whether to solve $G$ top-down or bottom-up — are related. In particular, bottomup reasoning can be interpreted as generating a model in which $G$ is true.

These issues are hard enough for Horn clause programs. But they are much harder for logic programs with negative conditions. In some semantics, a negative condition not $B$ has the same meaning as classical negation $\neg B$, and solving a negative goal not $B$ is interpreted as reasoning with $\neg B$. But in most proof theories, not $B$ is interpreted as some form of negation as failure:

not $B$ holds if all attempts to show $B$ fail.

In addition to these purely logical problems concerning semantics and proof theory, LP has been plagued by controversies concerning declarative versus procedural representations. Declarative representations are naturally supported by bottomup model generation. But both declarative and procedural representations can be supported by top-down execution. For many advocates of purely declarative representations, such exploitation of procedural representations undermines the logic programming ideal.

These issues of semantics, proof theory and knowledge representation have been a recurring theme in the history of LP, and they continue to be relevant today. They are reflected, in particular, by the growing divergence between Prolog-style systems that employ top-down execution and answer set programming and Datalog systems that employ bottom-up model generation.

\section{THE HISTORICAL BACKGROUND}

The discovery of the top-down method for executing logic programs occurred in the summer of 1972, as the result of my collaboration with Alain Colmerauer in Marseille. Colmerauer was developing natural language question-answering systems, and I was developing resolution theorem-provers, and trying to reconcile them with procedural representations of knowledge in artificial intelligence.

\subsection{Resolution}

Resolution was developed by John Alan Robinson [1965a] as a technique for automated theorem-proving, with a view to mechanising mathematical proofs. It consists of a single inference rule for proving that a set of assumptions $P$ logically implies a theorem $G$. The resolution method is a refutation procedure, which does so by reductio ad absurdum, converting $P$ and the negation $\neg G$ of the theorem into a set of clauses and deriving the empty clause, which represents falsity.

Clauses are disjunctions of literals. In Robinson's original definition, clauses were represented as sets. In the propositional case: 
given two clauses $\{A\} \cup F$ and $\{\neg A\} \cup G$

the resolvent is the clause $F \cup G$.

The two clauses $\{A\} \cup F$ and $\{\neg A\} \cup G$ are said to be the parents of the resolvent, and the literals $A$ and $\neg A$ are said to be the literals resolved upon. If $F$ and $G$ are both empty, then the resolvent of $\{A\}$ and $\{\neg A\}$ is the empty clause, representing a contradiction or falsity.

In the first-order case, in which all variables are universally quantified with scope the clause in which they occur, it is necessary to unify sets of literals to make them complementary:

given two clauses $K \cup F$ and $L \cup G$

the resolvent is the clause $F \theta \cup G \theta$.

where $\theta$ is a most general substitution of terms for variables that unifies the atoms in $K$ and $L$, in the sense that $K \theta=\{A\}$ and $L \theta=\{\neg A\}$. It is an important property of resolution, which greatly contributes to its efficiency, that if there is any substitution that unifies $K$ and $L$, then there is a most general such unifying substitution, which is unique up to renaming of variables.

The set representation of clauses (and sets of clauses) builds in the inference rules of commutativity, associativity and idempotency of disjunction (and conjunction). The resolution rule itself generalises modus ponens, modus tollens, disjunctive syllogism, and many other separate inference rules of classical logic. The use of the most general unifier, moreover, subsumes in one operation, the infinitely many inferences of the form "derive $P(t)$ from $\forall X P(X)$ " that are possible with the inference rule of universal instantiation. Other inference rules are eliminated (or used) in the conversion of sentences of standard first-order logic into clausal form.

Set notation for clauses is not user-friendly. It is more common to write clauses $\left\{A_{1}, \ldots, A_{n}, \neg B_{1}, \ldots, \neg B_{m}\right\}$ as disjunctions $A_{1} \vee \ldots \vee A_{n} \vee \neg B_{1} \vee \ldots \vee \neg B_{m}$. However, sets of clauses, representing conjunctions of clauses, are commonly written simply as sets. Clauses can also be represented as conditionals in the form:

$$
A_{1} \vee \ldots \vee A_{n} \leftarrow B_{1} \wedge \ldots \wedge B_{m}
$$

where $\leftarrow$ is material implication $\rightarrow$ (or $\supset$ ) written backwards.

The discovery of resolution revolutionised research on automated theorem proving, as many researchers turned their hands towards developing refinements of the resolution rule. It also inspired other applications of logic in artificial intelligence, most notably to the development of question-answering systems, which represent data or knowledge in logical form, and query that knowledge using logical inference. One of the most successful and most influential such system was QA3, developed by Cordell Green [1969].

In QA3, given a knowledge base and goal to be solved, both expressed in clausal form, an extra literal answer $(X)$ is added to the clause or clauses representing the negation of the goal, where the variables $X$ represent some value 
of interest in the goal. For example, to find the capital of the usa, the goal $\exists X \operatorname{capital}(X, u s a)$ is negated and the answer literal is added, turning it into the clause $\neg \operatorname{capital}(X, u s a) \vee$ answer $(X)$. The goal is solved by deriving a clause consisting only of answer literals. The substitutions of terms for variables used in the derivation determine values for the variables $X$. In this example, if the knowledge base contains the clause capital(washington, usa), the answer answer(washington) is obtained in one resolution step.

Green also showed that resolution has many other problem-solving applications, including robot plan formation. Moreover, he showed how resolution could be used to automatically generate a program written in a conventional programming language, such as LISP, from a specification of its input-output relation written in the clausal form of logic. As he put it:

"In general, our approach to using a theorem prover to solve programming problems in LISP requires that we give the theorem prover two sets of initial axioms:

1. Axioms defining the functions and constructs of the subset of LISP to be used

2. Axioms defining an input-output relation such as the relation $R(x, y)$, which is to be true if and only if $x$ is any input of the appropriate form for some LISP program and $y$ is the corresponding output to be produced by such a program."

Green also seems to have anticipated the possibility of dispensing with (1) and using only the representation (2) of the relation $R(x, y)$, writing:

"The theorem prover may be considered an 'interpreter' for a high-level assertional or declarative language - logic. As is the case with most high-level programming languages the user may be somewhat distant from the efficiency of 'logic' programs unless he knows something about the strategies of the system."

"I believe that in some problem solving applications the 'high-level language' of logic along with a theorem-proving program can be a quick programming method for testing ideas."

However, he does not seem to have pursued these ideas much further. Moreover, there was an additional problem, namely that the resolution strategies of that time behaved unintuitively and were very redundant and inefficient. For example, given a clause of the form $L_{1} \vee \ldots \vee L_{n}$, and $n$ clauses of the form $\neg L_{i} \vee C_{i}$, resolution would derive the same clause $C_{1} \vee \ldots \vee C_{n}$ redundantly in $n$ ! different ways.

\subsection{Procedural representations of knowledge}

Green's ideas fired the enthusiasm of researchers working in contact with him at Stanford and Edinburgh, but they also attracted fire from MIT, where researchers 
were advocating procedural representations of knowledge. Terry Winograd's PhD thesis gave the most compelling and most influential voice to this opposition. Winograd [1971] argued (page 232):

"Our heads don't contain neat sets of logical axioms from which we can deduce everything through a 'proof procedure'. Instead we have a large set of heuristics and procedures for solving problems at different levels of generality."

He quoted (pages 232-3) Green's own admission of some of the difficulties:

"It might be possible to add strategy information to a predicate calculus theorem prover, but with current systems such as QA3, 'To change strategies in the current version, the user must know about set-ofsupport and other program parameters such as level bound and term depth. To radically change the strategy, the user presently has to know the LISP language and must be able to modify certain strategy sections of the program.' (<Green 1969>p. 236)." 3

Winograd's procedural alternative to purely "uniform" logical representations was based on Carl Hewitt's language Planner. Winograd [1971] describes Planner in the following terms (page 238):

"The language is designed so that if we want, we can write theorems in a form which is almost identical to the predicate calculus, so we have the benefits of a uniform system. On the other hand, we have the capability to add as much subject-dependent knowledge as we want, telling theorems about other theorems and proof procedures. The system has an automatic goal-tree backup system, so that even when we are specifying a particular order in which to do things, we may not know how the system will go about doing them. It will be able to follow our suggestions and try many different theorems to establish a goal, backing up and trying another automatically if one of them leads to a failure (see section 3.3)."

In contrast (page 215):

"Most 'theorem-proving' systems do not have any way to include this additional intelligence. Instead, they are limited to a kind of 'working in the dark'. A uniform proof procedure gropes its way through the collection of theorems and assertions, according to some general procedure which does not depend on the subject matter. It tries to combine facts which might be relevant, working from the bottom-up."

\footnotetext{
${ }^{3}$ We will see later that the set-of-support strategy was critical, because it allowed QA3 to incorporate a form of backward reasoning from the theorem to be proved.
} 
Winograd's PhD thesis presented a natural language understanding system that was a great advance at the time, and its advocacy of Planner was enormously influential. Even Stanford and Edinburgh were affected by these ideas.

Pat Hayes and I had been working in Edinburgh on a book [Hayes and Kowalski, 1971] about resolution theorem-proving, when he returned from a second visit to Stanford (after the first visit, during which he and John McCarthy wrote the famous situation calculus paper [McCarthy and Hayes, 1968]). He was greatly impressed by Planner, and wanted to rewrite the book to take Planner into account. I was not enthusiastic, and we spent many hours discussing and arguing about the relationship between Planner and resolution theorem proving. Eventually, we abandoned the book, unable to agree.

\subsection{Resolution, part two}

At the time that QA3 and Planner were being developed, resolution was not well understood. In particular, it was not understood that a proof procedure, in general, is composed of an inference system that defines the space of all proofs and a search strategy that explores the proof space looking for a solution of a goal. We can represent this combination as an equation:

$$
\text { proof procedure }=\text { proof space }+ \text { search strategy }
$$

A typical proof space has the structure of an and-or tree turned upside down. Typical search strategies include breadth-first search, depth-first search and some form of best-first or heuristic search.

In the case of the resolution systems at the time, the proof spaces were horrendously redundant, and most search strategies used breadth-first search. Attempts to improve efficiency focussed on restricting (or refining) the resolution rule without losing completeness, to reduce the size of the proof space. The best known refinements were hyper-resolution and set of support.

Hyper-resolution [Robinson, 1965b] is a generalised form of bottom-up (or forward) reasoning. In the propositional case, given an input clause:

$$
D_{0} \vee \neg B_{1} \vee \ldots \vee \neg B_{m}
$$

and $m$ input or derived positive clauses:

$$
B_{1} \vee D_{1}, \ldots, B_{m} \vee D_{m}
$$

where each $B_{i}$ is an atom and each $D_{i}$ is a disjunction of atoms, hyper-resolution derives the positive clause:

$$
D_{0} \vee D_{1} \vee \ldots \vee D_{m}
$$

Bottom-up reasoning with Horn clauses is the special case in which $D_{0}$ is a single atom and each other $D_{i}$ is an empty disjunction, equivalent to false. In this special 
case, rewriting disjunctions as conditionals, hyper-resolution derives $B_{0}$ from the input clause:

$$
B_{0} \leftarrow B_{1} \wedge \ldots \wedge B_{m}
$$

and the input or derived facts, $B_{1}, \ldots, B_{m}$.

The problem with hyper-resolution, as Winograd observed, is that it derives new clauses from the input clauses, without paying attention to the problem to be solved. It is "uniform" in the sense that, given a theorem to be proved, it uniformly performs the same inferences bottom-up from the axioms, ignoring the theorem until it generates it, as if by accident.

In contrast with hyper-resolution, the set of support strategy [Wos et al., 1965] focuses on a subset of clauses that are relevant to the problem at hand:

A subset $S^{\prime}$ of an input set $S$ of clauses is a set of support for $S$ iff $S-S^{\prime}$ is satisfiable. The set of support strategy restricts resolution so that at least one parent clause belongs to the set of support or is derived by the set of support restriction.

If the satisfiable set of clauses $S-S^{\prime}$ represents a set of axioms, and the set of support $S^{\prime}$ represents the negation of a theorem, then the set of support strategy implements an approximation of top-down reasoning by reductio ad absurdum. It also ensures that any input clauses (or axioms) used in a derivation are relevant to the theorem, in the spirit of relevance logics [Anderson and Belnap, 1962]. ${ }^{4}$

The set of support strategy only approximates top-down reasoning. A better approximation is obtained by linear resolution, which was discovered independently by Loveland [1970], Luckham [1970] and Zamov and Sharonov [1969]. Linear resolution addresses the problem of relevance by focusing on a top clause $C_{0}$, which could represent an initial goal:

Let $S$ be a set of clauses. A linear derivation of a clause $C_{n}$ from a top clause $C_{0} \in S$ is a sequence of clauses $C_{0}, \ldots, C_{n}$ such that every clause $C_{i+1}$ is a resolvent of $C_{i}$ with some input clause in $S$ or with some ancestor clause $C_{j}$ where $j<i$. (It was later realised that ancestor resolution is unnecessary if $S$ is a set of Horn clauses.)

The top clause $C_{0}$ in a linear derivation can be restricted to one belonging to a set of support. The resulting space of all linear derivations from a given top clause $C_{0}$ has the structure of a proof tree whose nodes are clauses and whose branches are linear derivations. Using linear resolution to extend the derivation of a clause $C_{i}$ to the derivation of a clause $C_{i+1}$ generates the derived node $C_{i+1}$ as a child of

\footnotetext{
${ }^{4}$ Another important case is the one in which $S-S^{\prime}$ represents a database (or knowledge base) together with a set of integrity constraints that are satisfied by the database, and $S^{\prime}$ represents a set of updates to be added to the database. The set of support restriction then implements a form of bottom-up reasoning from the updates, to check that the updated database continues to satisfy the integrity constraints. Moreover, it builds in the assumption that the database satisfied the integrity constraints prior to the updates, and therefore if there is an inconsistency, the update must be "relevant" to the inconsistency.
} 
the node $C_{i}$. The same node $C_{i}$ can have different children $C_{i+1}$, corresponding to different linear resolutions.

In retrospect, the relationship with Planner is obvious. If the top clause $C_{0}$ represents an initial goal, then the tree of all linear derivations is a goal tree, and generating the tree top-down is a form of goal-reduction. The tree can be explored using different search strategies. Depth-first search, in particular, can be informed by Planner-like strategies that both specify "a particular order in which to do things", but also "back up" automatically in the case of failure.

The relationship with Planner was not obvious at the time. Even as recently as 2005, Paul Thagard in Mind: Introduction to Cognitive Science, compares logic unfavourably with production systems, stating on page 45 :

"In logic-based systems, the fundamental operation of thinking is logical deduction, but from the perspective of rule-based systems, the fundamental operation of thinking is search." 5

But it wasn't just this lack of foresight that stood in the way of understanding the relationship with Planner: there was still the $n$ ! redundant ways of resolving upon $n$ literals in the clauses $C_{i}$. This redundancy was recognized and eliminated without the loss of completeness by Loveland [1972], Reiter [1971], and Kowalski and Kuehner [1971], independently at about the same time. The obvious solution was simply to resolve upon the literals in the clauses $C_{i}$ in a single order. This order can be determined statically, by ordering the literals in the input clauses, and imposing the same order on the resolvents. Or it could be determined dynamically, as in selected linear (SL) resolution [Kowalski and Kuehner, 1971], by selecting a single literal to resolve upon in a clause $C_{i}$ when the clause is chosen for resolution. Both methods eliminate redundancy, but dynamic selection can lead to smaller search spaces. ${ }^{6}$

Ironically, both Loveland [1972] and Kowalski and Kuehner [1971] also noted that linear resolution with an ordering restriction is equivalent to Loveland's [1968] earlier model elimination proof procedure. The original model elimination procedure was presented so differently that it took years even for its author to recognise the equivalence. The SL resolution paper also pointed out that the set of all SL derivations forms a search space, and described a heuristic search strategy for finding simplest proofs. In the conclusions, with implicit reference to Planner, it claimed:

\footnotetext{
${ }^{5}$ This claim makes more sense if Thagard, like Winograd before him, associates logic exclusively with forward reasoning. As Sherlock Holmes explained to Dr. Watson, in A Study in Scarlet: "In solving a problem of this sort, the grand thing is to be able to reason backward. That is a very useful accomplishment, and a very easy one, but people do not practise it much. In the everyday affairs of life it is more useful to reason forward, and so the other comes to be neglected. There are fifty who can reason synthetically for one who can reason analytically."

${ }^{6}$ Dynamic selection is useful, for example, to solve goals with different input-output arguments. For example, given the clause $p(X, Y) \leftarrow q(X, Z) \wedge r(Z, Y)$ and the goal $p(a, Y)$, then the subgoal $q(a, Z)$ should be selected before $r(Z, Y)$. But given the goal $p(X, b)$, the subgoal $r(Z, b)$ should be selected before $q(X, Z)$.
} 
"Moreover, the amenability of SL-resolution to the application of heuristic methods suggests that, on these grounds alone, it is at least competitive with theorem-proving procedures designed solely from heuristic considerations."

\section{THE PROCEDURAL INTERPRETATION OF HORN CLAUSES}

The development of various forms of linear resolution with set of support and ordering restrictions brought resolution systems closer to Planner-like theoremprovers. But these resolution systems did not yet have an explicit procedural interpretation.

\subsection{The representation of grammars in logical form}

Among the various confusions that prevented a better understanding of the relationship between logical and procedural representations was the fact that Winograd's thesis, which so advanced the Planner cause, employed a different procedural language Programmar, for natural language grammars. Moreover, Winograd's natural language understanding system was implemented in a combination of micro-Planner (a subset of Planner), Programmar and LISP. So it wasn't obvious whether Planner was supposed to be a general-purpose programming language, or a special purpose language for proving theorems, for writing plans or for some other purpose.

In the theorem-proving group in Edinburgh, where I was working at the time, much of the debate surrounding Planner focused on whether "uniform", resolution proof procedures are adequate for proving theorems, or whether they need to be augmented with Planner-like, domain-specific control information. In particular, I was puzzled by the relationship between Planner and Programmar, and began to investigate whether grammars could be written in a logical form. This was auspicious, because in the summer of 1971 Alain Colmerauer invited me for a short visit to Marseille.

Colmerauer knew everything there was to know about formal grammars and their application to programming language compilers. During 1967-1970 at the University of Montreal, he developed Q-systems [1969] as a rule-based formalism for processing natural language. Q-systems were later used on a daily basis from 1982 to 2001 to translate English weather forecasts into French for Environment Canada. Since 1970, he had been in Marseille, building up a team working on natural language question-answering, investigating SL-resolution for the questionanswering component.

I arrived in Marseille, anxious to get Colmerauer's feedback on my preliminary ideas about representing grammars in logical form. My representation used a function symbol to concatenate words into strings of words, and axioms to express that concatenation is associative. It was obvious that reasoning with such associativity axioms was inefficient. Colmerauer immediately saw how to avoid the 
axioms of associativity, in a representation that later came to be known as metamorphosis grammars [Colmerauer, 1975] (or definite clause grammars [Pereira and Warren, 1980]). We saw that different kinds of resolution applied to the resulting grammars give rise to different kinds of parsers. For example, forward reasoning with hyper-resolution performs bottom-up parsing, while backward reasoning with SL-resolution performs top-down parsing. ${ }^{7}$

\subsection{Horn clauses and SLD-resolution}

It was during my second visit to Marseille in April and May of 1972 that the idea of using SL-resolution to execute Horn clause programs emerged. By the end of the summer, Colmerauer's group had developed the first version of Prolog, and used it to implement a natural language question-answering system [Colmerauer et al., 1973]. I reported an abstract of my own findings at the MFCS conference in Poland in August 1972 [Kowalski, 1972]. ${ }^{8}$

The first Prolog system was an implementation of SL-resolution for the full clausal form of first-order logic, including ancestor resolution. But the idea that Horn clauses were an interesting case was already in the air. Donald Kuehner [1969], in particular, had already been working on bi-directional strategies for Horn clauses. However, the first explicit reference to the procedural interpretation of Horn clauses appeared in [Kowalski, 1974]. The abstract begins:

"The interpretation of predicate logic as a programming language is based upon the interpretation of implications: $B$ if $A_{1}$ and. . and $A_{n}$ as procedure declarations, where $B$ is the procedure name and $A_{1}$ and ... and $A_{n}$ is the set of procedure calls constituting the procedure body."

The theorem-prover described in the paper is a variant of SL-resolution, to which Maarten van Emden later attached the name SLD-resolution, standing for "selected linear resolution with definite clauses":

A definite clause is a Horn clause of the form $B \leftarrow B_{1} \wedge \ldots \wedge B_{n}$.

A goal clause is a Horn clause of the form $\leftarrow A_{1} \wedge \ldots \wedge A_{n}$.

Given a goal clause $\leftarrow A_{1} \wedge \ldots \wedge A_{i-1} \wedge A_{i} \wedge A_{i+1} \wedge \ldots \wedge A_{n}$ with selected atom $A_{i}$ and a definite clause $B \leftarrow B_{1} \wedge \ldots \wedge B_{m}$, where $\theta$ is a most general substitution that unifies $A_{i}$ and $B$, the $S L D$-resolvent is the goal clause $\leftarrow\left(A_{1} \wedge \ldots \wedge A_{i-1} \wedge B_{1} \wedge \ldots \wedge B_{m} \wedge A_{i+1} \wedge \ldots \wedge A_{n}\right) \theta$

Given a set of definite clauses $S$ and an initial goal clause $C_{0}$, an $S L D$ derivation of a goal clause $C_{n}$ is a sequence of goal clauses $C_{0}, \ldots, C_{n}$

\footnotetext{
${ }^{7}$ However, Colmerauer [1991] remembers coming up with the alternative representation of grammars, not during my visit in 1971, but after my visit in 1972 .

${ }^{8}$ In the abstract, I used a predicate $\operatorname{val}(f(X), Y)$ instead of a predicate $f(X, Y)$, using Phillip Roussel's idea of val as "formal equality". Roussel was Colmerauer's PhD student and the main implementer of the first Prolog system.
} 
such that every $C_{i+1}$ is the SLD-resolvent of $C_{i}$ with some input clause in $S$.

An SLD-refutation is an SLD-derivation of the empty clause.

SLD-resolution is more flexible than SL-resolution restricted to Horn clauses. ${ }^{9}$ In SL-resolution the atoms $A_{i}$ must be selected last-in-first-out, but in SLDresolution, there is no restriction on their selection. Both refinements of linear resolution avoid the redundancy of unrestricted linear resolution, and both are complete, in the sense that if a set of Horn clauses is unsatisfiable, then there exists both an SL-resolution refutation and an SLD-resolution refutation in their respective search spaces. In both cases, different selection strategies give rise to different, complete search spaces. But the more flexible selection strategy of SLDresolution means that search spaces can be smaller, and therefore more efficient to search.

In SLD resolution, goal clauses have a dual interpretation. In the strictly logic interpretation, the symbol $\leftarrow$ in a goal clause $\leftarrow A_{1} \wedge \ldots \wedge A_{n}$ is equivalent to classical negation; the empty clause is equivalent to falsity; and a refutation indicates that the top clause is inconsistent with the initial set of clauses $S$.

However, in a problem-solving context, it is natural to think of the symbol $\leftarrow$ in a goal clause $\leftarrow A_{1} \wedge \ldots \wedge A_{n}$ as a question mark ? or command !, and the conjunction $A_{1} \wedge \ldots \wedge A_{n}$ as a set of subgoals, whose variables are all existentially quantified. The empty clause represents an empty set of subgoals, and a "refutation" indicates that the top clause has been solved. The solution is represented by the substitutions of terms for variables in the top clause, generated by the most general unifiers used in the refutation - similar to, but without the answer literals of QA3.

As in the case of linear resolution more generally, the space of all SLD-derivations with a given top clause has the structure of a goal tree, which can be explored using different search strategies. From a logical point of view, it is desirable that the search strategy be complete, so that the proof procedure is guaranteed to find a solution if there is one in the search space. Complete search strategies include breadth-first search and various kinds of best-first and heuristic search. Depthfirst search is incomplete in the general case, but it takes up much less space than the alternatives. Moreover, it is complete if the search space is finite, or if there is only one infinite branch that is explored after all of the others.

Notice that there are two different, but related notions of completeness: one for search spaces, and the other for search strategies. A search space is complete if it contains a solution whenever the semantics dictates that there is a solution; and a search strategy is complete if it finds a solution whenever there is one in the search space. For a proof procedure to be complete, both its search space and its search strategy need to be complete.

\footnotetext{
${ }^{9}$ If SL-resolution is applied to Horn clauses, with a goal clause as top clause, then ancestor resolution is not possible, because all clauses in the same SL-derivation are then goal clauses, which cannot be resolved with one another.
} 
The different options for selecting atoms to resolve upon in SLD-resolution and for searching the space of SLD-derivations were left open in [Kowalski, 1974], but were pinned down in the Marseille Prolog interpreter. In Prolog, subgoals are selected last-in-first-out in the order in which the subgoals are written, and branches of the search space are explored depth-first in the order in which the clauses are written. By choosing the order in which subgoals and clauses are written, a Prolog programmer can exercise considerable control over the efficiency of a program.

\subsection{Logic + Control}

In those days, it was widely believed that logic alone is inadequate for problemsolving, and that some way of controlling the theorem-prover is needed for efficiency. Planner combined logic and control in a procedural representation that made it difficult to identify the logical component. Logic programs with SLDresolution also combine logic and control, but make it possible to read the same program both logically and procedurally. I later expressed this as Algorithm = Logic + Control $(A=L+C)$ [Kowalski, 1979a], influenced by Pat Hayes ${ }^{\prime}[1973]$ Computation $=$ Controlled Deduction.

The most direct implication of the equation is that, given a fixed logical representation $L$, different algorithms can be obtained by applying different control strategies, i.e. $A_{1}=L+C_{1}$ and $A_{2}=L+C_{2}$. Pat Hayes [1973], in particular, argued that logic and control should be expressed in separate languages, with the logic component $L$ providing a pure, declarative specification of the problem, and the control component $C$ supplying the problem solving strategies needed for an efficient algorithm $A$. Moreover, he argued against the idea, expressed by $A_{1}=L_{1}+C$ and $A_{2}=L_{2}+C$, of using a fixed control strategy $C$, as in Prolog, and formulating the logic $L_{i}$ of the problem to obtain a desired algorithm $A_{i}$.

This idea of combining logic and control in separate object and meta-level languages has been a recurring theme in the theorem-proving and AI literature. It was a major influence, for example, on the development of PRESS, which solved equations by expressing the rules of algebra in an object language, and the rules for controlling the search for solutions in a meta-language. According to its authors, Alan Bundy and Bob Welham [1981]:

"PRESS consists of a collection of predicate calculus clauses which together constitute a Prolog program. As well as the procedural meaning attached to these clauses, which defines the behaviour of the PRESS program, they also have a declarative meaning - that is, they can be regarded as axioms in a logical theory."

In retrospect, PRESS was an early example of a now common use of Prolog to write meta-interpreters.

But most applications do not need such an elaborate combination of logic and control. For example, the meta-level control program in PRESS does not need a 
meta-meta-level control program. In fact, for some applications, even the modest control available to the Prolog programmer is unnecessary For these applications, it suffices for the programmer to specify only the logic of the problem, and to leave it to Prolog to solve the problem without any help

But often, leaving it to Prolog alone can result, not only in unacceptable inefficiency, but even in non-terminating failure to find a solution. Here is a simple example, written in Prolog notation, where :- stands for $\leftarrow$ and every clause ends in a full stop:

$$
\begin{aligned}
& \text { likes }(\text { bob }, X):-\operatorname{likes}(X, \operatorname{logic}) \\
& \text { likes }(\text { bob, logic }) \\
& :-\operatorname{likes}(\text { bob }, X) .
\end{aligned}
$$

Prolog fails to find the solution $X=b o b$, because it explores the infinite branch generated by repeatedly using the first clause, without getting a chance to explore the branch generated by the second clause. If the order of the two clauses is reversed, Prolog finds the solution. If only one solution is desired then it terminates. But if all solutions are desired, then it encounters the infinite branch, and goes into the same infinite loop. Perhaps the easiest way to avoid such infinite loops in ordinary Prolog is to write a meta-interpreter, as in PRESS. ${ }^{10}$

Problems and inefficiencies with the Prolog control strategy led to numerous proposals for LP languages incorporating enhanced control features. Some of them, such as Colmerauer's [1982] Prolog II, which allowed insufficiently instantiated subgoals to be suspended, were developed as extensions of Prolog. Other proposals that departed more dramatically from ordinary Prolog included the use of coroutining in IC-Prolog [Clark et al., 1972] selective backtracking [Bruynooghe and Pereira, 1984] and meta-level control for logic programs [Gallaire and Lasserre, 1982; Pereira, 1984]

IC-Prolog, in particular, led to the development by Clark and Gregory [1983, 1986] of the concurrent logic programming language Parlog, which led in turn to numerous variants of concurrent LP languages, one of which KL1, developed by Kazunori Ueda [1986], was adopted as the basis for the systems software of the Fifth Generation Computer Systems (FGCS) Project in Japan.

The FGCS Project was a ten year project beginning in 1982, sponsored by Japan's Ministry of International Trade and Industry and involving all the major Japanese computer manufacturers. Its main objective was to develop a new generation of computers employing massive parallelism and oriented towards artificial intelligence applications. From the start of the project, logic programming was identified as the preferred software technology.

\footnotetext{
${ }^{10}$ In other cases, much simpler solutions are often possible. For example, to avoid infinite loops with the program $\operatorname{path}(X, X)$ and $\operatorname{path}(X, Y) \leftarrow \operatorname{link}(X, Z) \wedge \operatorname{path}(Z, Y)$, it suffices to add an extra argument to the path predicate to record the list of nodes visited so far, and to add an extra condition to the second clause to check that the node $Z$ in $\operatorname{link}(X, Z)$ is not in this path. For some advocates of declarative programming this is considered cheating. For others, it illustrates a practical application of $A=L_{1}+C_{1}=L_{2}+C_{2}$.
} 
The FGCS project did not achieve its objectives, and all three of its main areas of research - parallel hardware, logic programming software, and AI applications - suffered a world-wide decline.

These days, however, there is growing evidence that the FGCS project was ahead of its time. In the case of logic programming, in particular, SLD-resolution extended with tabling [Tamaki and Sato, 1986; Sagonas et al., 1994; Chen and Warren, 1996; Telke and Liu, 2011] avoids many infinite loops, like the one in the example above. Moreover, there also exist alternative techniques for executing logic programs that do not rely upon the procedural interpretation, including the model generation methods of Answer Set programming (ASP) and the bottom-up execution strategies of Datalog.

ASP and Datalog have greatly advanced the ideal of purely declarative representations, relegating procedural representations to the domain of imperative languages and other formalisms of dubious character. However, not everyone is convinced that purely declarative knowledge representation is adequate either for practical computing or for modelling human reasoning.

Thagard [2005], for example, claims that the following, useful procedure cannot easily be expressed in logical terms (page 45):

If you want to go home and you have the bus fare, then you can catch a bus.

On the contrary, the sentence can be expressed literally in the logical form:

$$
\text { can }(\text { you,catch-bus }) \leftarrow \text { want }(\text { you,go-home }) \wedge \text { have }(\text { you,bus-fare })
$$

But this rendering requires the use of modal operators or modal predicates for want and can. More importantly, it misses the real logic of the procedure:

$$
\text { go }(\text { you, home }) \leftarrow \text { have }(\text { you, bus-fare }) \wedge \operatorname{catch}(\text { you,bus }) .
$$

Top-down reasoning applied to this logic generates the procedure, without sacrificing either the procedure or the declarative belief that justifies it

\section{THE SEMANTICS OF HORN CLAUSE PROGRAMS}

The earliest influences on the development of logic programming had come primarily from automated theorem-proving and artificial intelligence. But researchers in the School of AI in Edinburgh also had strong interests in the theory of computation, and there was a lot of excitement about Dana Scott's [1970] recent fixed point semantics for programming languages. Maarten van Emden suggested that we investigate the application of Scott's ideas to Horn clause programs and that we compare the fixed point semantics with the logical semantics.

\subsection{What is the meaning of a program?}

But first we needed to establish a common ground for the comparison. If we identify the data structures of a logic program $P$ with the set of all ground terms 
constructible from the vocabulary of $P$, also called the Herbrand universe of $P$, then we can view the "meaning" (or denotation) of $P$ as the set of all ground atoms $A$ that can be derived from $P^{11}$, which is expressed by:

$$
P \vdash A \text {. }
$$

Here $\vdash$ can represent any derivability relation. Viewed in programming terms, this is analogous to the operational semantics of a programming language. But viewed in logical terms, this is a proof-theoretic definition, which is not a semantics at all. In logical terms, it is more natural to understand the semantics of $P$ as given by the set of all ground atoms $A$ that are logically implied by $P$, written:

$$
P \vDash A
$$

The operational and model-theoretic semantics are equivalent for any sound and complete notion of derivation - the most important kinds being top-down and bottom-up.

Top-down derivations include model-elimination, SL-resolution and SLD-resolution. Model-elimination and SL-resolution are sound and complete for arbitrary clauses. So they are sound and complete for Horn clauses in particular. Moreover, ancestor resolution is impossible for Horn clauses. So model-elimination and SL-resolution without ancestor resolution are sound and complete for Horn clause programs.

The selection rule in both SL-resolution and SLD-resolution constructs a linear representation of an and-tree proof. In SL-resolution the linear representation is obtained by traversing the and-tree depth-first. In SLD-resolution the linear representation can be obtained by traversing the and-tree in any order. ${ }^{12}$ The completeness of SLD-resolution was first proved by Robert Hill [1974].

Bottom-up derivations are a special case of hyper-resolution, which is also sound and complete for arbitrary clauses, and therefore for Horn clauses as well. Moreover, as we soon discovered, they are equivalent to the fixed point semantics.

\subsection{Fixed point semantics}

In Dana Scott's [1970] fixed point semantics, the denotation of a recursive function is given by its input-output relation. The denotation is constructed by approximation, starting with the empty relation, repeatedly plugging the current approximation of the denotation into the definition of the function, transforming the approximation into a better one, until the complete denotation is obtained in the limit, as the least fixed point.

\footnotetext{
${ }^{11}$ Notice that this excludes programs which represent perpetual processes. Moreover, it ignores the fact that, in practice, logic programs can compute input-output relations containing variables. This is sometimes referred to as the "power of the logical variable".

${ }^{12}$ Note that and-or trees suggest other strategies for executing logic programs, for example by decomposing goals into subgoals top-down, searching for solutions of subgoals in parallel, then collecting and combining the solutions bottom-up. This is like the MapReduce programming model used in Google [Dean and Ghemawat, 2008].
} 
Applying the same approach to a Horn clause program $P$, the fixed point semantics uses a similar transformation $T_{P}$, called the immediate consequence operator, to map a set $I$ of ground atoms representing an approximation of the input-output relations of $P$ into a more complete approximation $T_{P}(I)$ :

$$
T_{P}(I)=\left\{A_{0} \mid A_{0} \leftarrow A_{1} \wedge \ldots \wedge A_{n} \in \operatorname{ground}(P) \text { and }\left\{A_{1}, \ldots, A_{n}\right\} \subseteq I\right\} .
$$

Here $\operatorname{ground}(P)$ is the set of all ground instances of the clauses in $P$ over the Herbrand universe of $P$. The application of $T_{P}$ to $I$ is equivalent to applying one step of hyper-resolution to the clauses in $\operatorname{ground}(P) \cup I$.

Not only does every Horn clause program $P$ have a fixed point $I$ such that $T_{P}(I)=I$, but it has a least fixed point, lfp $\left(T_{P}\right)$, which is the denotation of $P$ according to the fixed point semantics. The least fixed point is also the smallest set of ground atoms $I$ closed under $T_{P}$, i.e. the smallest set $I$ such that $T_{P}(I) \subseteq I$. This alternative characterisation provides a link with the minimal model semantics, as we will see below.

The least fixed point can be constructed, as in Scott's semantics, by starting with the empty set \{\} and repeatedly applying $T_{P}$ :

$$
\text { If } T_{P}^{0}=\{\} \text { and } T_{P}^{i+1}=T_{P}\left(T_{P}^{i}\right) \text {, then } \operatorname{lfp}\left(T_{P}\right)=\cup_{0 \leq i} T_{P}^{i} .
$$

The result of the construction is equivalent to the set of all ground atoms that can be derived by applying any number of steps of hyper-resolution to the clauses in ground $(P)$.

The equality $l f p\left(T_{P}\right)=\cup_{0 \leq i} T_{P}^{i}$ is usually proved in fixed point theory by appealing to the Tarski-Knaster theorem. However, in [van Emden and Kowalski, 1976], we showed that the equivalence follows from the completeness of hyperresolution and the relationship between least fixed points and minimal models. Here is a sketch of the argument:

$$
\begin{aligned}
& A \in \operatorname{lfp}\left(T_{P}\right) \text { iff } A \in \min (P) \\
& \text { i.e. least fixed points and minimal models coincide. } \\
& A \in \min (P) \text { iff } P \vDash A \\
& \text { i.e. truth in the minimal model and all models coincide. } \\
& P \vDash A \text { iff } A \in \cup_{0 \leq i} T_{P}^{i} \\
& \text { i.e. hyper-resolution is complete. }
\end{aligned}
$$

\subsection{Minimal model semantics}

The minimal model semantics was inspired by the fixed point semantics, but it was based on the notion of Herbrand interpretation. The key idea of Herbrand interpretations is to identify an interpretation of a set of sentences with the set of all ground atomic sentences that are true in the interpretation.

In a Herbrand interpretation, the domain of individuals is the set of ground terms in the Herbrand universe of the language. A Herbrand interpretation is any 
subset of the Herbrand base, which is the set of all ground atoms of the language. The most important property of Herbrand interpretations is that, in first-order logic, a set of sentences has a model if and only if it has a Herbrand model. This property is a form of the Skolem-Löwenheim-Herbrand theorem. ${ }^{13}$

Thus the model-theoretic denotation of a Horn clause program:

$$
M(P)=\{A \mid A \text { is a ground atom and } P \vDash A\}
$$

is actually a Herbrand interpretation of $P$ in its own right. Moreover, it is easy to show that $M(P)$ is also a Herbrand model of $P$. In fact, it is the smallest Herbrand model $\min (P)$ of $P$. Therefore:

$$
A \in \min (P) \text { iff } P \vDash A \text {. }
$$

It is also easy to show that the Herbrand models of $P$ coincide with the Herbrand interpretations that are closed under the operator $T_{P}$, i.e.:

$$
I \text { is a Herbrand model of } P \text { iff } T_{P}(I) \subseteq I \text {. }
$$

This is because the immediate consequence operator mimics, not only hyperresolution, but also the definition of truth for Horn clauses: A set of Horn clauses $P$ is true in a Herbrand interpretation $I$ if and only if, for every ground instance $A_{0} \leftarrow A_{1} \wedge \ldots \wedge A_{n}$ of a clause in $P, A_{0}$ is true in $I$ if $A_{1}, \ldots, A_{n}$ are true in $I$.

It follows that the least fixed point and the minimal model are identical:

$$
l f p\left(T_{P}\right)=\min (P) .
$$

\subsection{Computability}

The logicians Andréka and Németi visited Edinburgh in 1975, and wrote a report, published in [Andréka and Németi, 1978], proving the Turing completeness of Horn clause logic. Sten-Åke Tärnlund [1977] obtained a similar result independently. It was a great shock, therefore, to learn that Raymond Smullyan [1956] had already published an equivalent result. Here is the complete abstract:

A new approach to recursive enumerability is considered based on the notion of "minimal models". A formula of the lower functional calculus of the form $F_{1} \cdot F_{2} \cdots F_{n-1} \cdot \supset \cdot F_{n}$ (or $F_{1}$ alone, if $n=1$ ) in which each $F_{i}$ is atomic, and $F_{n}$ contains no predicate constants, is termed regular. Let $A$ be a finite set of regular formulae; $\Sigma$ a collection of

\footnotetext{
${ }^{13}$ The property can be proved in two steps: First, convert $S$ into clausal form by using "Skolem" functions to eliminate existential quantifiers. Although the resulting set $S^{\prime}$ of clauses and $S$ are not equivalent, $S$ has a model iff $S^{\prime}$ has a model. A set of clauses $S^{\prime}$ has a model iff $S^{\prime}$ has a Herbrand model $M$, constructed using the Herbrand universe of $S^{\prime}$. Therefore $S$ has a model if and only if it has a Herbrand model $M$. (Contrary claims in the literature that $S$ may have a model, but no Herbrand model, are based on the assumption that the Herbrand interpretations of $S$ are constructed using the Herbrand universe of $S$.)
} 
sets and relations, on some universe $U ; \quad I$ an interpretation of the predicate constants (occurring in $A$ ) as elements of $\Sigma$. The ordered triple $\mathcal{L}$ viz. $(A, U, I)$ is a recursive logic over $\Sigma$. A model of $\mathcal{L}$ is an interpretation of the predicate variables $P_{i}$ in which each formula of $A$ is valid. Let $P_{i}^{*}$ be the intersection of all attributes assignable to $P_{i}$ in some model; these $P_{i}^{*}$ are called definable in $\mathcal{L}$. If each $P_{i}$ is interpreted as $P_{i}^{*}$, it can be proved that there is a model — this is the minimal model. Sets definable in some $\mathcal{L}$ over $\Sigma$ are termed recursively definable from $\Sigma$. It is proved: (1) the recursively enumerable sets are precisely those which are recursively definable from the successor relation and the unit set $\{0\} ;(2)$ Post's canonical sets in an alphabet $a_{1} \cdots a_{n}$, are those recursively definable from the concatenation relation and the unit sets $\left\{a_{1}\right\} \cdots\left\{a_{n}\right\}$.

Smullyan seems not to have published the details of his proofs. But he investigated the relationship between derivability and computability in his book on the Theory of Formal Systems [Smullyan, 1961]. These formal systems are variants of the canonical systems of Post, with strong similarities to Horn clause programs.

\subsection{Logic and databases}

The question-answering systems of the 1960s and 1970s represented information in logical form, and used theorem-provers to answer questions represented in logical form. It was the application of SL-resolution to such deductive question-answering that led to Colmerauer's work on Prolog. In the meanwhile, Ted Codd [1970] published his relational model, which represented data as relations in logical form, but used the "non-deductive" algebraic operations of selection, projection, Cartesian product, set union and set difference, to specify database queries. However, he also showed [Codd, 1972] that the relational algebra is equivalent to a more declarative relational calculus, in which relations are defined in first-order logic.

I first learned about relational databases in 1974 at a course on the foundations of computer science at the Mathematics Centre in Amsterdam. I was giving a short course of lectures on logic for problem solving, using a set of notes, which I later expanded into my 1979 book [Kowalski, 1979b]. Erich Neuhold was giving a course about formal properties of databases, with a focus on the relational model. It was immediately obvious that the relational model and logic programming had much in common.

I organised a five day workshop at Imperial College London in May 1976, using the term "logic programming" to describe the topic of the workshop. A full day was devoted to presentations about logic and databases. Hervé Gallaire and JeanMarie Nicholas presented the work they were doing in Toulouse, and Keith Clark talked about his work on negation as failure.

Jack Minker visited Gallaire and Nicholas in 1976, and together they organised the first workshop on logic and databases in Toulouse in 1977. The proceedings of 
the workshop, published in 1978, included Clark's results on negation as failure, and Reiter's paper on closed world databases.

\section{NEGATION AS FAILURE - PART 1}

The practical value of extending Horn clause programs to normal logic programs with negative conditions was recognized from the earliest days of logic programming, as was the obvious way to reason with them - by negation as failure (abbreviated as NAF): to prove not $p$, show that all attempts to prove $p$ fail. Intuitively, NAF is justified by the assumption that the program contains a complete definition of its predicates. The assumption is very useful in practice, but was neglected in formal logic. The problem was to give this proof-theoretic notion a logical semantics.

Ray Reiter [1978] investigated NAF in the context of a first-order database $D$, interpreting it as the closed world assumption ( $C W A$ ) that the negation not $p$ of a ground atom $p$ holds in $D$ if there is no proof of $p$ from $D$. He showed that the CWA can lead to inconsistencies in the general case - for example, given the database $D=\{p \vee q\}$, it implies not $p$, and not $q$; but for Horn data bases no such inconsistencies can arise.

However, Keith Clark was the first to investigate NAF in the context of logic programs with negative conditions.

\subsection{The Clark completion}

Clark's solution was to interpret logic programs as short hand for definitions in if-and-only-if form, as illustrated for the propositional program in figure 2.

\begin{tabular}{|c|c|}
\hline$p \leftarrow q \wedge r$ & $p \leftrightarrow(q \wedge r) \vee(s \wedge t)$ \\
\hline$p \leftarrow s \wedge t$ & $r \leftrightarrow u \vee v$ \\
\hline$r \leftarrow u$ & $q \leftrightarrow$ true \\
\hline$r \leftarrow v$ & $v \leftrightarrow$ true \\
\hline$q$ & $s \leftrightarrow$ false \\
\hline \multirow[t]{2}{*}{$v$} & $t \leftrightarrow$ false \\
\hline & $u \leftrightarrow$ false \\
\hline
\end{tabular}

Figure 2. The logic program of figure 1, and its completion.

In the non-ground case, the logic program needs to be augmented with an equality theory, which mimics the unification algorithm, and which essentially 
specifies that ground terms are equal if and only if they are syntactically identical. An example with a fragment of the necessary equality theory, is given in figure 3. Together with the equality theory, the if-and-only-if form of a logic program $P$ is called the completion of $P$, written $\operatorname{comp}(P)$. It is also sometimes called the predicate completion or the Clark completion.

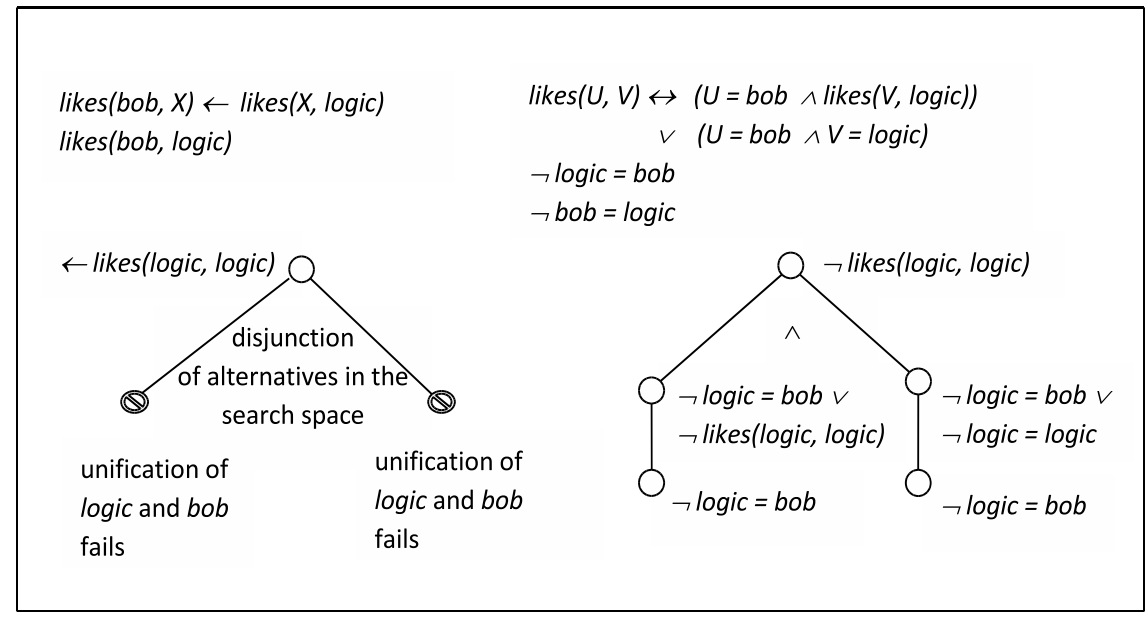

Figure 3. A proof of not likes(logic,logic) using negation as failure and backward reasoning compared with an upside down proof of $\neg$ likes(logic,logic) using classical logic. Notice that the use of classical negation turns the disjunction of alternatives into a logical conjunction.

As figure 3 illustrates, negation as failure correctly simulates reasoning with the completion in classical logic.

Although NAF is sound with respect to the completion semantics, it is not complete. For example, if $P$ is the program:

$$
\begin{aligned}
& p \leftarrow q \\
& p \leftarrow \neg q \\
& q \leftarrow q
\end{aligned}
$$

then $\operatorname{comp}(P)$ implies $p$. But given the goal $\leftarrow p, N A F$ goes into an infinite loop trying, but failing to show $q$. The completion semantics does not recognise such infinite failure, because proofs in classical logic are finite. For this reason, the completion semantics is also called the semantics of negation as finite failure.

In contrast with the completion semantics, the CWA formalises negation as potentially infinite failure, inferring $\neg q$ from the Horn clause database $q \leftarrow q$. Similarly, the minimal model semantics of Horn clauses concludes that $\neg q$ is true in the minimal model of the program $q \leftarrow q$.

Clark did not investigate the relationship between the completion semantics and the various alternative semantics of Horn clauses. Probably the first such 
investigation was by Apt and van Emden [1982], who showed, among other things, that if $P$ is a Horn clause program then:

$$
I \text { is a Herbrand model of } \operatorname{comp}(P) \text { iff } T_{P}(I)=I \text {. }
$$

Compare this with the property that $I$ is a Herbrand model of $P$ iff $T_{P}(I) \subseteq I$.

\subsection{The analogy with arithmetic}

Clark's 1978 paper was not the first to propose the completion semantics. [Clark and Tärnlund, 1977] proposed using the completion together with induction schemas on the structure of terms to prove program properties, by analogy with the use of induction in first-order Peano arithmetic.

Consider the Horn clause definition of append $(X, Y, Z)$, which holds when the list $Z$ is the concatenation of the list $X$ followed by the list $Y$ :

$$
\begin{aligned}
& \text { append }(n i l, X, X) \\
& \operatorname{append}(\operatorname{cons}(U, X), Y, \operatorname{cons}(U, Z)) \leftarrow \operatorname{append}(X, Y, Z)
\end{aligned}
$$

This is analogous to the definition of plus $(X, Y, Z)$, which holds when $X+Y=Z$ :

$$
\begin{aligned}
& \operatorname{plus}(0, X, X) \\
& \operatorname{plus}(s(X), Y, s(Z)) \leftarrow \operatorname{plus}(X, Y, Z)
\end{aligned}
$$

Here the successor function $s(X)$ represents $X+1$, as in Peano arithmetic.

These definitions alone are adequate for computing their denotations. More generally, they are adequate for solving any goal clause (which is an existentially quantified conjunction of atoms). However, to prove program properties expressed in the full syntax of first-order logic, the definitions need to be augmented with their completions and induction axioms. For example, the completion and induction over the natural numbers are both needed to show that the plus relation defined above is functional:

$$
\forall X Y U V[p l u s(X, Y, U) \wedge p \operatorname{plus}(X, Y, V) \rightarrow U=V]
$$

Similarly, to show that append is associative, the definition of append needs to be augmented both with the completion and induction over lists.

Because many program properties can be expressed in the logic programming sublanguage of first-order logic, it can be hard to distinguish between clauses that are needed for computation, and clauses that are emergent properties. A similar problem arises with deductive databases. As Nicolas and Gallaire [1978] observed, it can be hard to distinguish between clauses that define data, and integrity constraints that restrict data.

For real applications, these distinctions are essential. For example, without making these distinctions, a programmer can easily write a program that includes both the definition of append and the property that append is associative. The resulting logic program would be impossibly inefficient. 
The analogy with arithmetic helps to clarify the relationships between the different semantics of logic programs: It suggests that the completion augmented with induction schemas is like the first-order axioms for Peano arithmetic, and the minimal model is like the standard model of arithmetic. The fact that both notions of arithmetic have a place in mathematics suggests that both kinds of "semantics" also have a place in logic programming.

Interestingly, the analogy also works in the other direction. The fact that minimal models are the denotations of logic programs shows that the standard model of arithmetic has a syntactic core, which consists of the Horn clauses that define addition and multiplication. Martin Davis [1980] makes a similar point, but his core is essentially the Horn clause definitions of addition and multiplication augmented with the Clark Equality Theory:

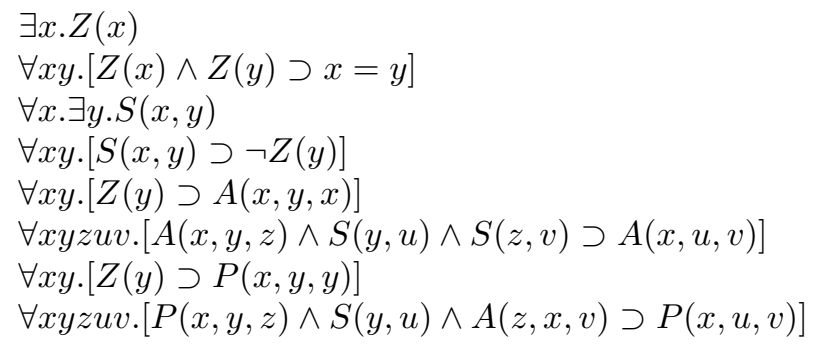

Here $Z(x)$ stands for " $x$ is zero", $S(x, y)$ for " $y$ is the successor of $x$ ", $A(x, y, z)$ for " $x+y=z$ " and $P(x, y, z)$ for " $x y=z$ ".

Arguably, the syntactic core of the standard model of arithmetic explains how we can understand what it means for a sentence to be true, even if we cannot prove that the sentence is true.

\subsection{Database semantics}

In the same workshop in which Clark presented his work, Nicolas and Gallaire [1978] considered related issues from a database perspective. They characterised the relational database approach as viewing databases as model-theoretic structures (or interpretations), and the deductive database approach as viewing databases as theories. They argued that, in relational databases, both query evaluation and integrity constraint satisfaction are understood as evaluating the truth value of a sentence in an interpretation. But in deductive databases, they are understood as determining whether the sentence is a theorem, logically implied by the database viewed as a theory. Hence the term "deductive". In retrospect, it is now clear that both kinds of databases, whether relational or "deductive", can be viewed either as an interpretation or as a theory.

A more fundamental issue at the time of the 1978 workshop was the inability of the relational calculus and relational algebra to define recursive relations, such as the transitive closure of a binary relation. Aho and Ullman [1979] proposed to remedy this by extending the relational algebra with fixed point operators. This 
proposal was pursued by Chandra and Harel [1982], who classified and analysed the complexity of the resulting hierarchy of query languages. Previously, Harel [1980] had published a harsh review of the logic and databases workshop proceedings [Gallaire and Minker, 1979], criticising it for claiming that deductive databases define relations in first-order logic despite the fact that transitive closure cannot be defined in first-order logic.

During the 1980s, the deductive database community, with roots mainly in artificial intelligence, became assimilated into a new Datalog community, influenced by logic programming, but with its roots firmly in the database field. In keeping with its database perspective, Datalog excludes function symbols. So all Herbrand models are finite, and are computable bottom-up. But pure bottom-up computation, whether viewed as model generation or as theorem-proving, ignores the query until it derives it as though by accident. To make model generation relevant to the query, Datalog uses transformations such as Magic Sets [Bancilhon, et al 1985] to incorporate the query into the transformed database rules.

As a consequence of its model generation approach, Datalog ignores the completion semantics in favour of the minimal model and fixed point semantics. For example, the surveys by Ceri, Gottlob and Tanca [1989], and Ramakrishnan and Ullman [1993], and even the more general survey of the complexity and expressive power of logic programming by Dantsin, Eiter, Gottlob and Voronkov [2001] mention the completion only in passing.

Minker's [1996] retrospective on Logic and Databases acknowledges the distinctive character of Datalog, but also includes the completion semantics. In particular, the completion semantics contributed to investigations of the semantics of integrity constraints, which was an important topic in deductive databases, before the field of Datalog fully emerged.

\section{NEGATION AS FAILURE - PART 2}

Theoretical investigations of the completion semantics continued, and were highlighted in John Lloyd's $[1985,1987]$ influential Foundations of Logic Programming book, which included results from Keith Clark's [1980] unpublished PhD thesis. Especially important among the later results were the three-valued completion semantics of Fitting [1985] and Kunen [1987], which gives, for example, the truth value undefined to $p$ in the program $p \leftarrow$ not $p$, whose completion is inconsistent in two-valued logic. This and other work on the completion semantics are presented in Shepherdson's [1988] survey. Much of this work concerns the correctness and completeness of SLDNF resolution (SLD resolution extended with negation as finite failure), relative to the completion semantics.

\subsection{Stratification}

The most significant next step in the investigation of negation was the study of stratified negation in database queries by Chandra and Harel [1985] and Naqvi 
[1986].

The simplest example of a stratified logic program is that of a deductive database $E \cup I$ whose predicates are partitioned into extensional predicates, defined by facts $E$, and intensional predicates, defined in terms of the extensional predicates by facts and rules $I$. Consider, for example, a network of nodes, some of whose links at any given time may be broken ${ }^{14}$. This can be represented by an extensional database, say:

$E: \quad \operatorname{link}(a, b) \quad \operatorname{link}(a, c) \quad \operatorname{link}(b, c) \quad \operatorname{broken}(a, c)$

Two nodes in the network are connected if there is a path of unbroken links. This can be represented intensionally by the clauses:

I: $\quad \operatorname{connected}(X, Y) \leftarrow \operatorname{link}(X, Y) \wedge \operatorname{not} \operatorname{broken}(X, Y)$

connected $(X, Y) \leftarrow \operatorname{connected}(X, Z) \wedge \operatorname{connected}(Z, Y)$

The conditions of the first clause in $I$ are completely defined by $E$. So they can be evaluated independently of $I$. The use of $E$ to evaluate these conditions results in a set of Horn clauses $I^{\prime}$, which intuitively has the same meaning as $I$ in the context of $E$ :

$$
\begin{array}{ll}
I^{\prime}: & \text { connected }(a, b) \quad \text { connected }(b, c) \\
& \text { connected }(X, Y) \leftarrow \operatorname{connected}(X, Z) \wedge \operatorname{connected}(Z, Y)
\end{array}
$$

The natural, intended model of the original deductive database $E \cup I$ is the minimal model $M$ of the resulting set of Horn clauses $E \cup I^{\prime}$ :

$$
\begin{array}{llll}
M: & \operatorname{link}(a, b) \quad \operatorname{link}(a, c) & \operatorname{link}(b, c) & \text { broken }(a, c) \\
& \text { connected }(a, b) \quad \text { connected }(b, c) & \text { connected }(a, c)
\end{array}
$$

This construction can be iterated if the intensional part of the database is also partitioned into layers (or strata). The further generalisation from databases to logic programs with function symbols was investigated independently by van Gelder [1989] and by Apt, Blair and Walker [1988].

Let $P$ be a logic program, and let Pred $=\operatorname{Pred}_{0} \cup \ldots \cup \operatorname{Pred}_{n}$ be a partitioning and ordering of the predicate symbols of $P$. If $A$ is an atomic formula, let $\operatorname{stratum}(A)=i$ if and only if the predicate symbol of $A$ is in $\operatorname{Pred}_{i}$. Then $P$ is stratified (with respect to this stratification of the predicate symbols), if and only if for every clause head $\leftarrow$ body in $P$ and for every condition $C$ in body:

if $C$ is an atomic condition, then $\operatorname{stratum}(C) \leq \operatorname{stratum}($ head)

if $C$ is a negative condition $\operatorname{not} A$, then $\operatorname{stratum}(A)<\operatorname{stratum}($ head).

\footnotetext{
${ }^{14}$ This example is inspired by the following quote from Hellerstein [2010]: "Classic discussions of Datalog start with examples of transitive closure on family trees: the dreaded anc and desc relations that afflicted a generation of graduate students. My group's work with Datalog began with the observation that more interesting examples were becoming hot topics: Web infrastructure such as webcrawlers and PageRank computation were essentially transitive closure computations, and recursive queries should simplify their implementation."
} 
The stratification Pred $=\operatorname{Pred}_{0} \cup \ldots \cup$ Pred $_{n}$ of the predicate symbols of $P$ induces a corresponding stratification of the program $P=P_{0} \cup \ldots \cup P_{n}$ where head $\leftarrow$ body in $P_{i}$ if and only if stratum $($ head $)=i$.

The perfect model of a stratified program $P$ is constructed by starting from the minimal model $M_{0}$ of the Horn clause program $P_{0}$ and iteratively extending the perfect model $M_{i-1}$ of $P_{0} \cup \ldots \cup P_{i-1}$ to the perfect model $M_{i}$ of $P_{0} \cup \ldots \cup P_{i}$. Assuming that the perfect model $M_{i-1}$ has already been constructed, then $M_{i}$ is constructed by using $M_{i-1}$ to evaluate the conditions in $P_{i}$ that are already defined in $P_{0} \cup \ldots \cup P_{i-1}$ obtaining a set of Horn clauses $P_{i}^{\prime}$, and generating $M_{i}$ as the minimal model of the Horn clauses $P_{i}^{\prime} \cup M_{i-1}$. Constructed in this way, $M_{n}$ is the perfect model of $P$.

For example, suppose we want to extend the logic program above by a clause that says a pair of nodes is unconnected if it is not connected:

$$
\text { unconnected }(X, Y) \leftarrow \text { not connected }(X, Y)
$$

The resulting program is stratified, with its predicates partitioned into the strata Pred $_{0}=\{$ link, broken $\}$, Pred $_{1}=\{$ connected $\}$, Pred $_{2}=\{$ unconnected $\}$. The perfect model $M_{2}$ of the program is constructed as follows:

$$
\begin{aligned}
M_{0}= & \{\operatorname{link}(a, b), \operatorname{link}(a, c), \operatorname{link}(b, c), \operatorname{broken}(a, c)\} \\
M_{1}= & M_{0} \cup\{\operatorname{connected}(a, b), \text { connected }(b, c), \text { connected }(a, c)\} \\
M_{2}= & M_{1} \cup\{\text { unconnected }(a, a), \text { unconnected }(b, b), \text { unconnected }(c, c), \\
& \text { unconnected }(b, a), \text { unconnected }(c, b), \text { unconnected }(c, a)\}
\end{aligned}
$$

It is useful to express the construction of perfect models using the notion of reduct, which relates it to the later construction of stable models by Gelfond and Lifschitz: Given a set of ground atoms $E$ and a logic program $P$, the reduct of $P$ by $E$, written $\operatorname{reduct}(P, E)$ is the set of Horn clauses obtained from $P$ by using $E$ to evaluate the negative literals in $P$ and using classical logic to simplify the resulting program. This construction is equivalent to deleting all clauses containing a condition not $B$ that is false in $E$ and deleting all conditions not $B$ that are true in $E$. Intuitively, if $E$ defines all of the non-atomic conditions in $P$, then $\operatorname{reduct}(P, E)$ has the same meaning as $P$ in the context of $E$. With this notion of reduct, the definition of perfect model can be expressed more concisely:

Given a stratified logic program $P=P_{0} \cup \ldots \cup P_{n}$, the perfect model of $P$ is $M_{n}$ where:

$$
\begin{aligned}
& M_{0}=\min \left(P_{0}\right) \\
& M_{i}=\min \left(\operatorname{reduct}\left(P_{i}, M_{i-1}\right) \cup M_{i-1}\right)
\end{aligned}
$$

Interestingly, this definition exploits the ambiguity of sets of atomic sentences, which can be viewed both as theories and as Herbrand interpretations. In its first occurrence in $\min \left(\right.$ reduct $\left.\left(P_{i}, M_{i-1}\right) \cup M_{i-1}\right), M_{i-1}$ is treated as a Herbrand interpretation. In its second occurrence, $M_{i-1}$ is treated as part of a Horn clause program. 


\subsection{Local stratification}

Przymusinski [1988] extended the notion of stratification from predicate symbols to ground atoms. In effect, this replaces a program $P$ by the program $\operatorname{ground}(P)$. In ground $(P)$, different atoms with the same predicate symbol are treated as distinct 0 -ary predicates, which can be assigned to different strata. Because of function symbols, ground $(P)$ can be countably infinite. Here is possibly the simplest sensible example that illustrates this:

$$
\text { Even: } \operatorname{even}(0) \quad \operatorname{even}(s(X)) \leftarrow \text { not even }(X))
$$

The program ground(Even) can be partitioned into a countably infinite number of subprograms ground $($ Even $)=\cup_{i<\omega}$ Even $_{i}$ where:

$$
\begin{array}{ll}
\text { Even }_{0}: & \operatorname{even}(0) \\
\text { Even }_{i}: & \operatorname{even}\left(s^{i}(0)\right) \leftarrow \operatorname{not} \operatorname{even}\left(s^{i-1}(0)\right) \text { for } i>0
\end{array}
$$

The perfect model is the limit $\cup_{i<\omega} M_{i}=\{\operatorname{even}(0)$, even $(s(s(0))), \ldots\}$ where:

$$
\begin{aligned}
& M_{0}=\min \left(\operatorname{Even}_{0}\right)=\{\operatorname{even}(0)\} \\
& M_{1}=\min \left(\operatorname{reduct}\left(\text { Even }_{1}, M_{0}\right) \cup M_{0}\right)=\min \left(\{\} \cup M_{0}\right)=M_{0} \\
& M_{2}=\min \left(\operatorname{reduct}\left(\operatorname{Even}_{2}, M_{1}\right) \cup M_{1}\right)=\min \left(\{\operatorname{even}(s(s(0)))\} \cup M_{0}\right) \\
& =\{\operatorname{even}(0), \operatorname{even}(s(s(0)))\} \\
& \text {... etc. }
\end{aligned}
$$

In general, let $P$ be a logic program, and let $H=\cup_{i<\alpha} H_{i}$ be a partitioning and ordering of the Herbrand base $H$ of $P$, where $\alpha$ is a countable, possibly transfinite ordinal. If $A \in H$, let $\operatorname{stratum}(A)=i$ if and only if $A \in H_{i}$. Then $P$ is locally stratified (with respect to this stratification of $H$ ) if and only if for every clause head $\leftarrow$ body in ground $(P)$ and for every condition $C$ in body:

if $C$ is an atomic condition, then $\operatorname{stratum}(C) \leq \operatorname{stratum}($ head) if $C$ is a negative condition $\operatorname{not} A$, then $\operatorname{stratum}(A)<\operatorname{stratum}($ head).

The stratification $\cup_{i<\alpha} H_{i}$ of $H$ induces a corresponding stratification of $\operatorname{ground}(P)=$ $\cup_{i<\alpha} P_{i}$ where head $\leftarrow$ body is in $P_{i}$ if and only if stratum(head) $=i$. The perfect model of $P$ is $M_{\alpha}$ where:

$$
\begin{aligned}
& M_{0}=\min \left(P_{0}\right) \\
& M_{i}=\min \left(\operatorname{reduct}\left(P_{i}, M_{i-1}\right) \cup M_{i-1}\right) \\
& M_{\beta}=\cup_{i<\beta} M_{i} \text { if } \beta \text { is a limit ordinal. }
\end{aligned}
$$

Unfortunately, although this construction gives the intended model for many natural programs, like Even above, it can fail even for minor syntactic variants of those programs. For example:

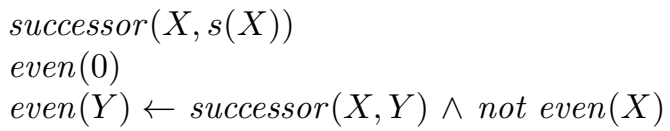


This program cannot be locally stratified, because its ground instances contain such unstratifiable clauses as even $(0) \leftarrow \operatorname{successor}(0,0) \wedge$ not even $(0)$.

Having recognised the problem, a number of authors proposed further refinements of stratification. However, it now seems to be generally agreed that these refinements are superseded by the well-founded semantics of [Van Gelder, Ross and Schlipf 1991]. In particular, [Denecker et al., 2001] argues that the well-founded semantics "provides a more general and more robust formalization of the principle of iterated inductive definition that applies beyond the stratified case."

\subsection{Well-founded semantics}

[Denecker et al., 2001] presents a simplified definition of the well-founded semantics in terms of candidate proofs. Here is a further simplification, in which candidate proofs are viewed as arguments supported by sets $\Delta$ of assumptions that are negative literals:

Given a ground normal program $P$, an argument for an atom $p$ supported by assumptions $\Delta$ is a finite tree $T$ labelled with literals such that:

- $p$ is the root of $T$.

- Each non-leaf node $q$ of $T$ is the head of some clause $q \leftarrow B$ in $P$, and its children are the literals in the body $B$ of the clause. If $q$ is a fact, with an empty body $B$, then it has a single child labelled by true.

- Each leaf is either the label true or a negative literal contained in $\Delta$.

Arguments can be used to construct a three-valued Herbrand model $M$ of $P$, represented by the set of all ground literals that are true in $M$. In general a threevalued Herbrand interpretation $I=I^{\text {pos }} \cup I^{\text {neg }}$ is a set of ground literals, such that every atom $A$ in $I^{\text {pos }}$ is true in $I$, and every atom $A$ whose negation not $A$ is in $I^{\text {neg }}$ is false in $I$. No atom $A$ is both true and false, and an atom $A$ that is neither true nor false is undefined.

The well-founded model can be generated bottom-up, starting with the empty set \{\} and repeatedly applying a three-valued consequence operator $\operatorname{Con}_{P}$, which extends a partial three-valued interpretation $I$ of $P$ to a more complete threevalued interpretation:

$\operatorname{Con}_{P}(I)=\left\{p \mid\right.$ there exists an argument for $p$ supported by $\left.I^{\text {neg }}\right\} \cup$ $\left\{\right.$ not $p \mid$ every argument for $p$ has a leaf not $q$ with $\left.q \in I^{\text {pos }}\right\}$

The well-founded model of $P$ is the smallest three-valued interpretation $I$ such that $\operatorname{Con}_{P}(I) \subseteq I$.

The well-founded model can also be queried top-down using SLG resolution [Chen and Warren, 1996], which is a variant of SLDNF with tabling. We will see later that the well-founded semantics also has an intuitive argumentation-theoretic interpretation, in which the negative literals not $p$ in $\operatorname{Con}_{P}(I)$ are all the assumptions defended by $I$. 
[Denecker, 1998] argued that the well-founded semantics formalizes the informal notion of inductive definition. In particular, the survey by [Denecker et al., 2001] of theories of inductive definitions in mathematical logic identifies two main approaches: inflationary inductive definitions and iterated inductive definitions. The abstract version of inflationary induction was investigated by Moschovakis [1974], and iterated induction was introduced by Kreisel [1963] and studied by Feferman [1970] and others.

Van Gelder [1993] observed that even simple concepts can be difficult to express using inflationary induction. For example, the complement of the transitive closure of a graph can be defined simply by a stratified logic program (as in the definition of unconnected in terms of connected). But it was considered a significant achievement when a solution was found using inflationary induction. [Denecker et al., 2001] argued that, in contrast, the well-founded semantics builds on the same principle as iterated inductive definitions, but is "more general and more robust".

\subsection{Stable model semantics}

Whereas the development of the well-founded semantics was influenced by stratification in deductive databases, the development of the stable model semantics [Gelfond and Lifschitz, 1988] was influenced by the problem of formalising default reasoning.

The earliest attempts to formalise default reasoning in artificial intelligence employed non-logical, object-oriented representations, such as semantic networks and frames [Minsky, 1975]. The first workshop dealing with logic-based approaches was held at Stanford in November 1978. A special issue of the Artificial Intelligence journal on non-monotonic reasoning, based on the workshop, was published in 1980. It contained papers by John McCarthy [1980] on circumscription, Ray Reiter [1980] on default logic, and Drew McDermott and Jon Doyle [1980] on nonmonotonic modal logic. It was later shown that circumscription and default logic have interesting relationships with the semantics of negation in logic programming.

Robert Moore [1985] developed a simple and elegant reconstruction of nonmonotonic modal logic, which was used later by Michael Gelfond [1987] to give an autoepistemic interpretation to normal logic programs. In Gelfond's translation, a logic program of the form $p \leftarrow q \wedge$ not $r$, for example, is translated into the sentence $p \leftarrow q \wedge \neg L r$ of autoepistemic logic, where $L r$ means $r$ is believed. Gelfond and Lifschitz [1988] further simplified this translation in the stable model semantics, interpreting a negative literal not $p$ as meaning that $p$ is not believed, in effect because the assumption that $p$ is not believed correctly leads to the conclusion that $p$ is not believed.

In general, given a normal logic program $P$ :

a Herbrand interpretation $M$ of $P$ is a stable model of $P$ iff $M=\min (\operatorname{reduct}(\operatorname{ground}(P), M))$.

For example, given $P=\{q, \quad p \leftarrow q \wedge$ not $r\}$, and the Herbrand interpretation 
$M=\{p, q\}$, the condition not $r$ is true in $M$, and therefore the set of Horn clauses $\operatorname{reduct}(P, M)=\{q, \quad p \leftarrow q\}$ has the same meaning as $P$ in the context of $M$. This meaning is the minimal model of $\operatorname{reduct}(P, M)$, which is identical to $M$. Therefore $M$ is a stable model of $P$. Moreover, it is the only stable model of $P$.

A program can have one, many or no stable models. For example the program $\{p \leftarrow$ not $p\}$ has no stable models, but $\{p \leftarrow$ not $q, q \leftarrow$ not $p\}$ has two stable models, $\{p\}$ and $\{q\}$.

The stable model semantics is non-monotonic, because adding clauses to a program can non-monotonically decrease the consequences of the program. For example, adding $r$ to the program $\{q, \quad p \leftarrow q \wedge$ not $r\}$ changes the minimal model from $\{p, q\}$ to $\{q\}$.

The stable model semantics has been extended to programs of the form:

$$
D_{1} \vee \ldots \vee D_{l} \leftarrow A_{1} \wedge \ldots \wedge A_{n} \wedge \text { not } B_{1} \wedge \ldots \wedge \text { not } B_{m}
$$

where $l \geq 0, n \geq 0$ and $m \geq 0$. Here each $D_{i}, A_{i}$ and $B_{i}$ is an atomic formula or the "explicit" negation $\neg A$ of an atomic formula $A$. If $l=0$, then the conclusion is equivalent to false.

The use of explicit negation (also called strong, or even classical negation) enables more natural knowledge representation, including the representation of rules and exceptions. For example:

$$
\begin{aligned}
& \operatorname{canfly}(X) \leftarrow \operatorname{bird}(X) \wedge \operatorname{not} \neg \operatorname{canfly}(X) \\
& \neg \operatorname{canfly}(X) \leftarrow \operatorname{penguin}(X)
\end{aligned}
$$

In the extended stable model semantics, explicit negations $\neg A$ are treated as syntactic sugar for "contrary" positive atoms, say $A^{*}$. For example, the literal $\neg \operatorname{canfly}(X)$ can be renamed as a positive atom, say abnormal $(X)$. With this renaming and the addition of clauses $\leftarrow A \wedge A^{*}$ for every pair of contrary atoms, the stable models of a program with explicit negation are isomorphic to the stable models of the same program without explicit negation.

Arguably, the extension to disjunctive conclusions is more problematic, because it creates the need to decide between alternative representations, for example between the two representations:

$$
\begin{aligned}
& p \leftarrow \operatorname{not} q \\
& p \vee \operatorname{not} q
\end{aligned}
$$

In the general case, different representations have different stable models.

In any case, the extension of the stable model semantics to include disjunctive conclusions clearly distances it from the stratified and well-founded semantics, which are naturally understood as the semantics of inductive definitions. The primer by Eiter et al., [2009], which presents a comprehensive survey of the stable model semantics and its associated Answer Set Programming paradigm, refers to the difference between these two kinds of semantics as the Great Logic Programming Schism. 
It might be more appropriate to call this the Second Great Schism, with the First Great Schism being between two different views of what it means for a logic program $P$ to solve a goal $G$. In the theorem-proving view, solving $G$ means showing that $P$ (or $\operatorname{comp}(P)$ ) logically implies $G$. But both in the stratified/wellfounded semantics and in the stable model semantics, solving $G$ means showing that $G$ is true in some canonical model of $P$.

\subsection{Answer set programming}

In the stratified/well-founded semantics, the intended model $M$ of a program is unique, the goal $G$ typically contains (existentially quantified) variables, and a solution of $G$ is a substitution $\sigma$ of terms for variables such that $G \sigma$ is true in $M$. In the paper that introduced the stable model semantics, Gelfond and Lifschitz [1988] similarly viewed the purpose of the stable model semantics as identifying a unique (or canonical) model of a program, writing:

"The stable model semantics is defined for a logic program $\Pi$, if $\Pi$ has exactly one stable model, and it declares that model to be the canonical model of $\Pi$."

According to this view, programs that have multiple stable models do not have a semantics at all.

The answer set programming (ASP) paradigm, proposed independently by Ilkka Niemelä [1999] and by Victor Marek and Miroslaw Truszczyński [1999] turns this point of view upside down. In ASP, the program itself is the problem to be solved, a stable model of the program is a solution, and different stable models are different solutions.

One of the simplest and most typical ASP examples is the map colouring problem. Given a map with countries $X$ represented by the predicate country $(X)$ and adjacent countries $X$ and $Y$ represented by adjacent $(X, Y)$, the problem is to find a colouring red, yellow or green for each country, such that no two adjacent countries have the same colour. Here, ignoring the constraint that no country should have two different colours, is a simple representation:

$$
\begin{aligned}
& \operatorname{colour}(X, \text { red }) \vee \operatorname{colour}(X, \text { yellow }) \vee \operatorname{colour}(X, \text { green }) \leftarrow \operatorname{country}(X) \\
& \leftarrow \operatorname{colour}(X, C) \wedge \operatorname{colour}(Y, C) \wedge \operatorname{adjacent}(X, Y)
\end{aligned}
$$

Clearly, the first clause can be rewritten as three normal clauses with negative conditions, in this case without affecting the stable models of the program. The second clause, which implicitly has the conclusion false, is a constraint, which excludes models that satisfy the conditions of the clause.

ASP is almost certainly the most active area of research in logic programming today. Because, for practical applications, solutions (and therefore stable models) need to be finite, it is common to restrict programs $P$ to ones whose grounding $\operatorname{ground}(P)$ is finite. To ensure finite groundings, ASP programs are often restricted to ones without function symbols, as in Datalog. For this reason, and because ASP 
and Datalog both employ bottom-up problem solving methods, the two areas have much in common.

\section{ABDUCTIVE LOGIC PROGRAMMING}

ASP also overlaps with constraint logic programming (CLP) and abductive logic programming (ALP). In a recent ASP programming competition [Denecker et al., 2009], competitors included one CLP system and three ALP systems. The top two systems were both ASP solvers, but an ALP-like solver achieved a respectable third place.

\subsection{Abduction}

Abduction was identified by the logician and philosopher Charles Sanders Peirce (1839-1914) as a form of logical reasoning, comparable to, but distinct from, deduction and induction. Whereas deduction derives conclusions from assumptions (e.g. $p(a)$ from $p(X) \leftarrow q(X)$ and $q(a)$ ), and induction derives general rules from facts (e.g. $p(X) \leftarrow q(X)$ from $p(a), p(b)$ and $q(a)$ ), abduction derives assumptions from rules and conclusions (e.g. $q(a)$ from $p(X) \leftarrow q(X)$ and $p(a))$. As the last example shows, abduction is closely related to top-down reasoning in logic programming.

Peirce's notion of abduction has had a big influence on epistemology and the philosophy of science, and inspired numerous applications in artificial intelligence. In my case, it was one of the inspirations of the concluding chapter of my 1979 book. It also inspired the development of Theorist [Poole et al., 1987] and the application of abduction to default reasoning.

In Poole [1988], abduction is used to extend a first-order clausal theory $T$ with assumptions $\Delta$ from a set of candidate hypotheses $A$, restricted by a set of firstorder constraints $I$ :

Given $T, A, I$ and observations $G$, an abductive explanation of $G$ is a subset $\Delta$ of $A$, such that

$$
\begin{aligned}
& T \cup \Delta \vDash G \\
& T \cup \Delta \cup I \text { is consistent. }
\end{aligned}
$$

The implementation of Theorist used a combination of linear resolution to generate candidate $\Delta$, by reasoning backwards from $G$, and a refutation proof procedure to show that $T \cup \Delta \cup I$ is consistent, by failing to refute $T \cup \Delta \cup I$. Although such a procedure is not even semi-decidable in theory, it is often sufficient in practice.

\subsection{Horn clause ALP and the relationship with stable models}

[Eshghi, 1988] and [Eshghi and Kowalski, 1989] reformulated Theorist in a logic programming setting, defining an abductive framework as a triple $\langle P, I, A\rangle$, where 
$P$ is a Horn clause program, $I$ is a set of integrity constraints, and $A$ is a set of ground atoms (whose predicates are called abducible):

Given an abductive framework $\langle P, I, A\rangle$ and a set $G$ of goal clauses, an abductive solution (or explanation) of $G$ is a subset $\Delta$ of $A$, such that

$$
\begin{aligned}
& P \cup \Delta \text { solves } G \\
& P \cup \Delta \text { satisfies } I .
\end{aligned}
$$

The requirement that $P \cup \Delta$ solves $G$ was defined as in Theorist, namely as $P \cup \Delta \vDash G$. But because $P \cup \Delta$ is a set of Horn clauses, it can also be defined as $G$ being true in the minimal model of $P \cup \Delta$. For integrity constraints $I$ in the form of denials $\leftarrow A_{1} \wedge \ldots \wedge A_{n}$, the requirement that $P \cup \Delta$ satisfies $I$ was also defined as in Theorist, namely as $P \cup \Delta \cup I$ is consistent.

Whereas Poole [1988] investigated a translation of default logic into Theorist, Eshghi and Kowalski [1989] investigated a translation of the stable model semantics into ALP. The translation treats the set of all ground negative literals as a set $\mathrm{Neg}$ of abducible atoms. In doing so, it treats a normal $\operatorname{logic} \operatorname{program} P$ with negative conditions as a Horn clause program $P^{*}$. It uses integrity constraints $I$ to ensure that ordinary atoms $a$ and their abducible negations not $a$ are complementary. This translates $P$ into an ALP program $\left\langle P^{*}, I, N e g\right\rangle$. The correspondence is given by the relationship:

Let $H$ be the Herbrand base of a normal logic program $P$.

Let $M \subseteq H$ and $\Delta \subseteq N e g$.

Then $M$ is a stable model of $P$ if and only if $P^{*} \cup \Delta$ satisfies $I$.

The integrity constraints $I$ needed for this correspondence include both all the denials $\leftarrow a \wedge$ not $a$ and all the disjunctions $a \vee$ not $a$. But at the time, we did not realise that the disjunctive constraints could be represented so simply. Instead, we represented them in a meta-logical form, in the spirit of Reiter's [1988] epistemic, modal representation of integrity constraints. I will come back to this problem of representing disjunctive constraints in the next subsection, 7.3.

Although [Eshghi and Kowalski, 1989] showed how to translate stable models into ALP, many ASP programs can be represented in ALP directly without the translation. For example, the map colouring program of section 6.5 can be represented by the ALP framework $\langle P, I, A\rangle$ where:

$P$ contains the definitions of the predicates country and adjacent.

$A$ is the predicate colour.

$I$ is the denial and disjunctive clause of the ASP program.

[Satoh and Iwayama, 1991] showed that the correspondence between stable models and ALP also works in the opposite direction: Let $\langle P, I, A\rangle$ be an ALP framework with denial integrity constraints $I$. For every $a \in A$, let not- $a$ be a distinct atom not occurring in $P$. Let $A^{*}$ be the set of all clauses: 


$$
\begin{aligned}
& a(X) \leftarrow \operatorname{not} \operatorname{not}-a(X) \\
& \operatorname{not}-a(X) \leftarrow \operatorname{not} a(X)
\end{aligned}
$$

Then $M$ is a stable model of $P \cup I \cup A^{*}$ if and only if $P \cup \Delta \vDash I$

\subsection{The ALP tower of Babel}

The definition of an ALP framework $\langle P, I, A\rangle$ given in section 7.2 can be generalized so that $P$ is a normal logic program with negation. But then the requirements that $P \cup \Delta$ solves $G$ and $P \cup \Delta$ satisfies $I$ have even more interpretations than before. In particular, the requirement that $P \cup \Delta$ solves $G$ can be interpreted either as $\operatorname{comp}(P \cup \Delta) \vDash G$, or as $G$ is true in some appropriate canonical model of $P \cup \Delta$.

The requirement that $P \cup \Delta$ satisfies $I$ is even more problematic. The problem was already the subject of extensive debate in the 1980s in the context of deductive databases $D$. The alternatives included the interpretation that the constraints $I$ are consistent with $D$ [Kowalski, 1978], that they are consistent with $\operatorname{comp}(D)$ [Sadri and Kowalski, 1988], that they are logically implied by $\operatorname{comp}(D)$ [Reiter, 1984; 1988; Lloyd and Topor, 1985], and that they are epistemic sentences that are true in $D$ [Reiter, 1988].

In the context of ALP, the different interpretations of solving a goal are multiplied by the different interpretations of satisfying integrity constraints. Compared with the stable model semantics and ASP, where everyone speaks with one voice, in ALP everyone argues in a different language. So is there any prospect of clearing up the confusion?

We can start by eliminating the semantic distinction between goals and integrity constraints. Arguably, the distinction is mainly a pragmatic one between goals $G$ that are one off (or ad hoc) and goals $I$ that are persistent and need to be maintained. As a consequence, the definition of abductive solution can be simplified:

Given an abductive framework $\langle P, I, A\rangle$ and a set $G$ of goal clauses, an abductive solution is a subset $\Delta$ of $A$, such that $P \cup \Delta$ satisfies $G \cup I$.

The hard part of the problem remains: How to understand integrity satisfaction? Having been involved in the early debates about the semantics of integrity constraints, and contributed to proof procedures for both integrity checking [Sadri and Kowalski, 1988] and ALP [Fung and Kowalski, 1997], I am now convinced that the requirement that $P \cup \Delta$ satisfies $G \cup I$ is best understood as $G \cup I$ is true in some appropriate model $M$ of $P \cup \Delta$. This interpretation has the added attraction that, no matter how $M$ is defined, $G \cup I$ can include arbitrary first-order sentences. In particular, $I$ can include the disjunctive constraints $a \vee$ not $a$, needed to simulate the stable model semantics, as was shown by Kakas and Mancarella [1990].

It remains to identify the nature of the model $M$. In the case of Horn clause programs $P$, it is obvious that $M$ should be the minimal model of $P \cup \Delta$. Similarly, in the case of locally stratified programs, $M$ should be the perfect model of $P \cup \Delta$. 
But if $P$ is an arbitrary normal logic program, then it is not immediately obvious whether $M$ should be a stable model or some canonical model, such as the wellfounded model.

However, the correspondence between the stable model semantics and abduction shows that stable models and abduction are different ways of achieving the same functionality. Allowing $M$ to be any stable model would be double counting. This leaves only one sensible alternative, namely restricting $M$ to some canonical model, with the well-founded model being the strongest candidate. The resulting combination of logic programs $P \cup \Delta$ defining well-founded models $M$ in which first-order sentences $G \cup I$ are true is closely related to the combination of firstorder logic with inductive definitions developed by Marc Denecker [1998] and his colleagues [Denecker et al., 2001]. The argument for understanding abduction in terms of the well-founded semantics was also made, from the viewpoint of representing default and hypothetical reasoning, by [Pereira et al., 1991].

\section{CONSTRAINT LOGIC PROGRAMMING}

Proof procedures for ALP and Constraint Logic Programming (CLP) have much in common: Both generate a set (or conjunction) $C$ of conditions (abducible or constraint formulas) such that $C$ solves the initial goal $G$ and $C$ is satisfiable. In both cases, they do so by reasoning top-down, backwards from $G$, incrementally generating $C$ and testing $C$ for satisfiability.

Constraints were first introduced into LP by Colmerauer [1982] in Prolog II. Their introduction was motivated mainly by the inefficiency of the "occur check" need to ensure that a term, such as $f(X)$, does not unify with a subterm, such as $X$. Clark [1978] had shown that unification with the occur check implements the identity relation for the domain $D$ of Herbrand interpretations, in which ground terms can be viewed as finite trees. Colmerauer [1982] showed that unification without the occur check implements the identity relation for the domain $D$ in which terms can be viewed as possibly infinite, rational trees.

Jaffar and Lassez [1987] introduced the CLP Scheme, which generalized the domain $D$ to an arbitrary model-theoretic structure defining the semantics of constraint predicates. The resulting programs are sets of Horn clauses whose bodies contain both user-defined predicates and constraint predicates, but whose heads contain only user-defined predicates. The most important new instance of the scheme was CLP $(R)$ [Jaffar et al., 1992], in which the constraint domain is the set of real numbers with addition, multiplication, identity and inequality.

The semantics of a CLP program [Jaffar et al., 1998] is given both by a structure $D$ and a theory $T$, which is a first-order axiomatization of $D$. The relationship between $D$ and $T$ is analogous to the relationship between the standard model of arithmetic and first-order Peano arithmetic.

The "algebraic" semantics is defined in terms of truth in the minimal model of $P \cup D$, where $D$ is the set of all ground constraint atoms that are true in $D$. 
According to the algebraic semantics, given a constraint logic program $P$, goal clause $G$ and constraint formula $C$ :

$G$ is satisfiable iff $G$ is true in the minimal model of $P \cup D$.

$C$ solves $G$ iff $C$ and $C \rightarrow G$ are true in the minimal model of $P \cup D$.

According to the "logical" semantics:

$G$ is satisfiable iff $P \cup T \vDash G$.

$C$ solves $G$ iff $P \cup T \vDash C \rightarrow G$ and $C$ is consistent with $P \cup T$.

According to the operational semantics:

$C$ solves $G$ iff $C$ can be generated by reasoning backwards from $G$, and $C$ is satisfiable, where satisfiability is determined by a constraint solver solve $(C)$, which may also simplify $C$.

There is an obvious parallel here, not only with proof procedures for ALP, but also with the two main alternative ways of defining the semantics of ALP.

The "algebraic" and "logical" semantics of the CLP Scheme coincide for Horn clause programs and theories $T$ that satisfy certain natural completeness conditions. But, in the case of CLP programs with negation, the relationship between the two kinds of semantics is much more problematic, but seems to have received relatively little attention.

If all these problems and confusions about the semantics of negation are not enough, there is one more twist to the story.

\section{ARGUMENTATION}

The proof procedure in the [Eshghi and Kowalski, 1989] paper was intended to compute the stable model semantics, but failed to implement the disjunctive integrity constraint in its totality. Phan Minh Dung [1991] showed that it implemented instead a localized form of the disjunctive constraint, to which he and [Kakas et al., 1992] gave an argumentation interpretation. Dung [1993, 1995] generalized this interpretation and developed an abstract argumentation theory, which has wide-ranging applications beyond logic programming.

In the case of logic programming, given a ground normal program $P$, an argument for a claim $p$ supported by assumptions $\Delta \subseteq N e g$ is a finite tree $T$ labelled with literals such that:

- $p$ is the root of $T$.

- Each non-leaf node $q$ of $T$ is the head of some clause $q \leftarrow B$ in $P$, and its children are the literals in the body $B$ of the clause. If $q$ is a fact, with an empty body $B$, then it has a single child labelled by true.

- Each leaf is either the label true or an assumption in $\Delta$. 
Viewed abstractly, it is the set $\Delta$ of assumptions supporting an argument that determines whether the argument and its claim are acceptable, and this depends, in turn, on whether or not $\Delta$ is able to defend itself against attack. Given a ground normal program $P$ :

$\Delta_{1} \subseteq N e g$ attacks $\Delta_{2} \subseteq N e g$ iff there exist an argument for a claim $p$ supported by $\Delta_{1}$ and an assumption not $p \in \Delta_{2}$.

$\Delta_{1} \subseteq N e g$ defends $\Delta_{2} \subseteq N e g$ iff

$\Delta_{1}$ attacks every $\Delta_{3} \subseteq N e g$ that attacks $\Delta_{2}$.

The notions of argument, assumption, attack and defence are sufficient to reconstruct not only most logic programming semantics, but also most semantics for non-monotonic reasoning [Bondarenko et al., 1997]. In the case of logic programming, sets $\Delta \subseteq N e g$ of assumptions that do not attack themselves correspond to three-valued Herbrand interpretations $M=M^{\text {pos }} \cup M^{\text {neg }}$ whose true atoms $M^{p o s}$ are supported by their false atoms $M^{n e g}=\Delta$. If $\Delta$ also contains all the assumptions that it defends, then the corresponding interpretation is a three-valued stable model, as defined by Przymusinski [1990] and shown by [Wu et al., 2009], and therefore a partial stable model, as defined by Sacca and Zaniolo [1990, 1991].

Sets $\Delta$ that not only contain all the assumptions that they defend, but also attack all the assumptions that they do not contain, correspond to two-valued, stable models:

Given a ground logic program $P$ :

If $\Delta \subseteq N e g$ does not attack $\Delta$, and $\Delta$ attacks $N e g-\Delta$, then $M=\{p \mid \Delta$ supports an argument for $p\}$ is a stable model of $P$.

If $M$ is a stable model of $P$, then

$\Delta=\{$ not $p \in N e g \mid p \notin M\}$ does not attack $\Delta$, and $\Delta$ attacks $N e g-\Delta$.

From an argumentation point of view, the stable model semantics is all-out warfare: For a set of assumptions $\Delta$ to correspond to a stable model, every assumption not $p$ has to take a side: Either not $p$ is in $\Delta$, or $\Delta$ attacks $\{$ notp $\}$.

Dung $[1993 ; 1995]$ argued that the stable model semantics is too extreme: It is sufficient for a set of assumptions to defend itself against all attacks:

$\Delta \subseteq N e g$ is admissible iff $\Delta$ defends $\Delta$, and $\Delta$ does not attack $\Delta$.

Dung [1991] showed that the proof procedure of [Eshghi and Kowalski, 1989] is sound with respect to the admissibility semantics.

Dung also gave an abductive interpretation of the well-founded semantics in [Dung, 1991] and an argumentation interpretation in [Dung, 1995]. Whereas in the admissibility and stable semantics an assumption can be used in its own selfdefense, in the well-founded semantics an assumption has to be defended by other assumptions:

A set $\Delta$ of assumptions is well-founded iff $\Delta$ is the smallest set of assumptions that contains all the assumptions that it defends. 
This is similar to Przymusinski's [1990] characterization of the well-founded semantics in terms of three-valued stable models.

The well-founded set can be constructed bottom-up by starting with the empty set \{\} of assumptions and repeatedly adding new assumptions defended by the previously added set of assumptions, until no further assumptions can be added. This bottom-up construction is similar to van Gelder's [1993] alternating fixed point characterisation of the well-founded semantics.

\section{CONCLUSIONS}

This history covers some of the highlights of the development of logic programming from the late $1960 \mathrm{~s}$ into the $21^{\text {st }}$ century. It focuses on a number of issues that are still relevant today, in particular on:

- the difference between solving a goal by theorem-proving and solving it by model generation,

- the difference between solving a goal top-down and solving it bottom-up.

- the relationship between declarative and procedural representations.

Perhaps the biggest change over the years has been the move away from viewing computation as deduction to viewing it as model generation. The seeds of this change were planted with the minimal model semantics of Horn clauses in 1976, but really got going only in the $1980 \mathrm{~s}$, when it was applied to the semantics of negation as failure. As a consequence, except perhaps in the context of CLP, the completion semantics of negation has been overshadowed by the model-theoretic approach.

The recent revival of Datalog [Huang et al., 2011] suggests that the old promise that logic programming can unify programming and databases may have new prospects. However, the query evaluation strategies of Datalog are mainly bottomup with magic set transformations used to simulate top-down execution. Is this simulation of top-down execution really necessary? Or might some more direct combination of top-down and bottom-up execution be more useful.

Recent years have also seen a shift away from reconciling declarative and procedural representations to a more purely declarative approach. In the meanwhile, imperative languages dominate the world of practical computing. Does this mean that logic programming is destined to become a niche technology concerned exclusively with declarative representations, based on ASP and Datalog? Or will it split into separate declarative and procedural camps, with procedural representations being relegated to the domain of Prolog? Or might it still be possible to reconcile declarative and procedural representations, perhaps by combining Prolog-like top-down execution with tabling? 


\section{ACKNOWLEDGEMENTS}

Many thanks to Maurice Bruynooghe, Keith Clark, Marc Denecker, Phan Minh Dung, Maarten van Emden, Michael Gelfond, Tony Kakas, Vladimir Lifschitz, Luis Pereira, Alan Robinson, John Schlipf, Jörg Siekmann, Allen Van Gelder and David Scott Warren for their helpful comments on the paper. Special thanks to Luis and Maarten for acting as official readers of the paper.

It is important to stress that, in this case more than in most, the author alone is fully responsible for any errors of fact or judgement.

\section{BIBLIOGRAPHY}

[Anderson and Belnap, 1962] A. R. Anderson and N. D. Belnap. The pure calculus of entailment. Journal of Symbolic Logic, 19-52, 1962.

[Andréka and Németi, 1978] H. Andréka and I. Németi. The Generalized Completeness of Horn Predicate Logic as a Programming Language. Acta Cybernetica, 4:3-10, 1978. (This is the publish version of a 1975 report entitled "General Completeness of Prolog" Department of Artificial Intelligence, University of Edinburgh).

[Apt and Bol, 1994] K. R. Apt and R. Bol. Logic programming and negation: a survey. Journal of Logic Programming 19-20, 9-71, 1994.

[Apt et al., 1988] K. R. Apt, H. Blair, and A. Walker. Towards a Theory of Declarative Knowledge. In: J. Minker (ed.), Foundations of Deductive Databases and Logic Programming, pp. 89-148. Morgan Kaufman, Los Altos, CA, 1988.

[Apt and van Emden, 1982] K. R. Apt and M. van Emden. Contributions to the Theory of Logic Programming. Journal of the ACM 29, 3, 841-862, 1982.

[Bancilhon et al., 1985] F. Bancilhon, D. Maier, Y. Sagiv, and J. D. Ullman. Magic sets and other strange ways to implement logic programs. In Proceedings of the fifth ACM SIGACTSIGMOD symposium on Principles of database systems (pp. 1-15). ACM, 1985.

[Bondarenko et al., 1997] A. Bondarenko, P. M. Dung, R. Kowalski, and F. Toni. An Abstract Argumentation-theoretic Approach to Default Reasoning. Journal of Artificial Intelligence 93 (1-2), 63-101, 1997.

[Boyer and Moore, 1972] R. S. Boyer and J. S. Moore. The sharing of structure in theoremproving programs. Machine Intelligence, 7, 101-116, 1972.

[Brewka et al., 2011] G. Brewka, T. Eiter, and M. Truszczyński. Answer set programming at a glance. Communications of the ACM, 54(12), 92-103, 2011.

[Bruynooghe and Pereira, 1984] M. Bruynooghe and L. M. Pereira. Deduction revision through intelligent backtracking. In J. Campbell, ed., "Issues in Prolog Implementation". Ellis Horwood, 1984.

[Bry et al., 2007] F. Bry, N. Eisinger, T. Eiter, T. Furche, G. Gottlob, C. Ley, and F. Wei Foundations of rule-based query answering. In Proceedings of the Third international summer school conference on Reasoning Web (pp. 1-153). Springer-Verlag, 2007.

[Bundy and Welham, 1981] A. Bundy and B. Welham. Using meta-level inference for selective application of multiple rewrite rule sets in algebraic manipulation. Artificial Intelligence, 16(2), 189-211, 1981.

[Ceri et al., 1990] S. Ceri, G. Gottlob, and L. Tanca. Logic Programming and Databases. Surveys in Computer Science. Springer-Verlag, 1990.

[Chandra and Harel, 1982] A. K. Chandra and D. Harel. Structure and complexity of relational queries. Journal of Computer and System Sciences 25, 1 (Aug.), 99-128, 1982.

[Chandra and Harel, 1985] A. K. Chandra and D. Harel. Horn clause queries and generalizations. Journal of Logic Programming 2, 1 (April), 1-15, 1985. A preliminary version of this paper, "Horn Clauses and the Fixpoint Query Hierarchy," appeared in the ACM Symp. on Principles of Database Systems, 1982.

[Chen and Warren, 1996] W. Chen and D. Warren. Tabled Evaluation with Delaying for General Logic Programs. JACM 43, 20-74, 1996. 
[Clark, 1978] K. L. Clark/ Negation by failure. In Gallaire, H. and Minker, J. [eds], Logic and Databases, Plenum Press, 293-322, 1978.

[Clark, 1980] K. L. Clark. Predicate logic as a computational formalism (Doctoral dissertation, Queen Mary, University of London), 1980.

[Clark and Gregory, 1983] K. L. Clark and S. Gregory). Parlog: A parallel logic programming language. Imperial College of Science and Technology Department of Computing, 1983.

[Clark and Gregory, 1986] K. L. Clark and S. Gregory. Parlog: Parallel programming in logic. ACM Transactions on Programming Languages and Systems (TOPLAS), 8(1), 1-49, 1986.

[Clark et al., 1982] K. L. Clark, F. G. McCabe, and S. Gregory. IC-Prolog language features, Logic programming, ed. Clark/Tarnlund, 254-266, 1982.

[Clark and Tärnlund, 1978] K. L. Clark and S.-A. Tärnlund. A first-order theory of data and programs. In Proceedings of the IFIP Congress 77, 939-944, 1978.

[Codd, 1970] E. F. Codd. Relational Completeness of Data Base Sublanguages. Database Systems: $65-98,1970$.

[Codd, 1972] E. F. Codd. Relational completeness of data base sublanguages (pp. 65-98). IBM Corporation, 1972.

[Cohen, 1988] J. Cohen. A View of the Origins and Development of Prolog, Communications ACM, vol 31, pp 26-36, 1988.

[Colmerauer, 1969] A. Colmerauer. Les systèmes Q ou un formalisme pour analyser et synthétiser des phrases sur ordinateur. Mimeo, Montréal, 1969.

[Colmerauer, 1970] A. Colmerauer. Total Precedence Relations, Journal ACM 1970, vol 17, pp 14-30, 1970.

[Colmerauer, 1975] A. Colmerauer. Les grammaires de metamorphose, Groupe d'Intelligence Artificielle, University de Marseille-Luminy (November 1975). Appears as 'Metamorphosis Grammars" in: L. Bolc (Ed.), Natural Language Communication with Computers, (Springer, 1978).

[Colmerauer, 1982] A. Colmerauer. Prolog II: Reference manual and theoretical model. Groupe D'intelligence Artificielle, Faculté Des Sciences De Luminy, Marseille, 1982.

[Colmerauer et al., 1973] A. Colmerauer, H. Kanoui, R. Pasero, and P. Roussel. Un système de communication homme-machine en français. Research report, Groupe d'Intelligence Artificielle, Université d'Aix-Marseille II, Luminy, 1973.

[Colmerauer and Roussel, 1996] A. Colmerauer and P. Roussel. The birth of Prolog. In History of programming languages-II (pp. 331-367). ACM, 1996.

[Console et al., 1991] L. Console, D. Theseider Dupre, and P. Torasso. On the relationship between abduction and deduction. Journal of Logic and Computation 2(5) 661-690, 1991.

[Costantini, 2002] S. Costantini. Meta-reasoning: A Survey. In Kakas, A.C., Sadri, F. (Eds.): Computational Logic: Logic Programming and Beyond. Springer Verlag. Vol. 2. 253-288, 2002.

[Chen and Warren, 1996] W. Chen and D. Warren. Tabled evaluation with delaying for general logic programs. JACM 43, 1 (January), 20-74, 1996.

[Dantsin et al., 1997] E. Dantsin, T. Eiter, G. Gottlob, and A. Voronkov. Complexity and expressive power of logic programming. In Proceedings of the Twelfth Annual IEEE Conference on Computational Complexity, June 24-27, 1997, Ulm, Germany, pp. 82-101. IEEE Computer Society Press, 1997

[Davis, 1980] M. Davis. The mathematics of non-monotonic reasoning. Artificial Intelligence, $13(1), 73-80,1980$.

[Dean and Ghemawat, 2008] J. Dean and S. Ghemawat. MapReduce: simplified data processing on large clusters. Communications of the ACM, 51(1), 107-113, 2008

[Denecker, 1998] M. Denecker. The well-founded semantics is the principle of inductive definition. In Logics in Artificial Intelligence, J. Dix, L. Farinas del Cerro, and U. Furbach, Eds. Lecture Notes in Artificial Intelligence, vol. 1489. Springer-Verlag, 1-16, 1998.

[Denecker et al., 2001] M. Denecker, M. Bruynooghe, and V. Marek. Logic programming revisited: logic programs as inductive definitions. ACM Transactions on Computational Logic, 2(4), 623-654, 2001.

[Denecker and Kakas, 2002] M. Denecker and A. Kakas. Abduction in logic programming. In Computational Logic: Logic Programming and Beyond (pp. 402-436). Springer Berlin Heidelberg, 2002. 
[Denecker et al., 2009] M. Denecker, J. Vennekens, S. Bond, M. Gebser, and M. Truszczynski. The second Answer Set Programming competition. In LPNMR, E. Erdem, F. Lin, and T. Schaub, Eds. LNCS, vol. 5753. Springer, 637-654, 2009

[Dung, 1991] P. M. Dung. Negation as hypothesis: an abductive foundation for logic programming. Proc. 8th International Conference on Logic Programming. MIT Press, 1991.

[Dung, 1993] P. M. Dung. On the acceptability of arguments and its fundamental roles in nonmonotonic reasoning and logic programming, Proceedings of IJCAI 1993, pp. 852-857, Morgan Kaufmann, 1993.

[Dung, 1995] P. M. Dung. On the acceptability of arguments and its fundamental role in nonmonotonic reasoning, logic programming and n-person games. Artificial intelligence, 77(2), 321-357, 1995

[Eiter et al., 2009] T. Eiter, G. Ianni, and T. Krennwallner. Answer set programming: A primer. In Reasoning Web. Semantic Technologies for Information Systems (pp. 40-110). Springer Berlin Heidelberg, 2009.

[Elcock, 1990] E. W. Elcock. Absys: The First Logic Programming Language - a Retrospective and a Commentary. Journal of Logic Programming, 9(1), 1-17, 1990.

[van Emden, 2006] M. van Emden. The Early Days of Logic Programming: A Personal Perspective. The Association of Logic Programming Newsletter, Vol. 19 n. 3, August 2006. http://www.cs.kuleuven.ac.be/textasciitildedtai/projects/ALP/newsletter/aug06/

[van Emden and Kowalski, 1976] M. van Emden and R. Kowalski. The Semantics of Predicate Logic as a Programming Language JACM , Vol. 23, No. 4, 733-742. 1976. Earlier version DCL Memo. School of Artificial Intelligence, University of Edinburgh (1974)

[Eshghi and Kowalski, 1989] K. Eshghi and R. Kowalski. Abduction Compared with Negation by Failure. In Sixth International Conference on Logic Programming, (eds. G. Levi and M. Martelli) MIT Press, 234-254, 1989.

[Feferman, 1970] S. Feferman. Formal theories for transfinite iterations of generalised inductive definitions and some subsystems of analysis. In Intuitionism and Proof theory, A. Kino, J. Myhill, and R. Vesley, Eds. North Holland, 303-326, 1970.

[Fitting, 1985] M. Fitting. A Kripke-Kleene semantics for logic programs*. The Journal of Logic Programming, 2(4), 295-312, 1985.

[Fung and Kowalski, 1997] T. H. Fung and R. Kowalski. The IFF Proof Procedure for Abductive Logic Programming. Journal of Logic Programming, 1997.

[Gallaire and Minker, 1978] H. Gallaire and J. Minker. Logic and Data Bases. Plenum Press, New York, 1978

[Gallaire and Lasserre, 1982] H. Gallaire and C. Lasserre. Metalevel control for logic programs.Logic Programming, 173-185, 1982.

[Gelernter, 1963] H. Gelernter. Machine generated problem solving graphs. In Proc. Symp, Math. Theory of Automata (pp. 179-203), 1963.

[Gelfond, 1987] M. Gelfond. On Stratified Autoepistemic Theories. In Proc. of AAAI87. Morgan Kaufman, 207-211, 1987.

[Gelfond and Lifschitz, 1988] M. Gelfond and V. Lifschitz. The stable model semantics for logic programming. In: Proceedings of the Fifth International Conference on Logic Programming (ICLP), 1070-1080, 1988

[Gelfond and Lifschitz, 1991] M. Gelfond and V. Lifschitz. Classical negation in logic programs and disjunctive databases. New Generation Computing 9, 3-4, 365-386, 1991.

[Green, 1969] C. Green. Application of theorem proving to problem solving. Proceedings of the 1st International Joint Conference on Artificial Intelligence. Morgan Kaufmann. 219-239, 1996.

[Harel, 1980] D. Harel. Review on Logic and Data Bases, Computing Reviews \#36,671 (Aug 1980), 367-369.

[Hayes, 1973] P. J. Hayes. Computation as Deduction, Proceedings 2nd MFCS Symposium, Czechoslovakia Academy of Sciences, Prague, Czechoslovakia, 1973.

[Hayes and Kowalski, 1971] P. J. Hayes and R. A. Kowalski. Lecture Notes on Automatic Theorem-Proving. DCL Memo 40. School of Artificial Intelligence, University of Edinburgh, 1971.

[Hellerstein, 2010] J. M. Hellerstein. The Declarative Imperative: Experiences and Conjectures in Distributed Logic, SIGMOD Record 39(1), 2010.

[Hewitt, 1971] C. Hewitt. Procedural Embedding of Knowledge In Planner. Proceedings of the $2^{\text {nd }}$ International Joint Conference on Artificial Intelligence. Morgan Kaufmann, 1971. 
[Hewitt, 2009] C. Hewitt. Middle History of Logic Programming: Resolution, Planner, Edinburgh LCF, Prolog, Simula, and the Japanese Fifth Generation Project, 2009. arXiv preprint arXiv:0904.3036

[Hill, 1974] R. Hill. LUSH Resolution and its Completeness. DCL Memo 78. School of Artificial Intelligence, University of Edinburgh, 1974.

[Huang et al., 2011] S. S. Huang, T. J. Green, and B. T. Loo. Datalog and Emerging Applications: an Interactive Tutorial. Proceedings of the 2011 ACM SIGMOD International Conference on Management of Data pp. 1213-1216, 2011

[Jaffar and Lassez, 1987] J. Jaffar and J. L. Lassez. Constraint logic programming. In Proceedings of the 14th ACM SIGACT-SIGPLAN symposium on Principles of programming languages (pp. 111-119). ACM, 1987.

[Jaffar et al., 1998] J. Jaffar, M. Maher, K. Marriott, and P. Stuckey. The semantics of constraint logic programs1. The Journal of Logic Programming, 37(1-3), 1-46, 1998.

[Jaffar et al., 1992] J. Jaffar, S. Michaylov, P. Stuckey, and R. H. Yap The CLP(R) language and system. ACM Transactions on Programming Languages and Systems (TOPLAS), 14(3), 339-395, 1992.

[Kakas and Mancarella, 1990] A. C. Kakas and P. Mancarella. Generalized Stable Models: A Semantics for Abduction. In ECAI (Vol. 90, pp. 385-391), 1990.

[Kakas et al., 1992] A. C. Kakas, R. Kowalski, and F. Toni. Abductive logic programming. Journal of Logic and Computation, 2(6), 719-770, 1992.

[Kakas et al., 1998] A. C. Kakas, R. Kowalski, and F. Toni. The Role of Logic Programming in Abduction, Handbook of Logic in Artificial Intelligence and Programming 5, Oxford University Press, 235-324, 1998 .

[Kowalski, 1970] R. Kowalski. Search strategies for theorem proving. Machine Intelligence, 5, 181-201, 1970

[Kowalski, 1972] R. Kowalski. The Predicate Calculus as a Programming Language (abstract). Procedings of the First MFCS Symposium, Jablonna, Poland, 1972.

[Kowalski, 1974] R. Kowalski. Predicate logic as a programming language. In Proceedings of IFIP 1974 [Stockholm, Sweden). North-Holland, Amsterdam, 1974, 569-574. This is the published version of DCL Memo 70, School of Artificial Intelligence, Univ. of Edinburgh, U.K., Nov. 1973.

[Kowalski, 1978] R. Kowalski. Logic for data description, in: H. Gallaire and J. Minker (eds.), Logic and Data Bases, Plenum Press, New York, pp. 77-103, 1978.

[Kowalski, 1979a] R. Kowalski. Algorithm = Logic+ Control. CACM, 22(7), 424-436, 1979.

[Kowalski, 1979b] R. Kowalski. Logic for Problem Solving. North Holland Elsevier, 1979. This is an expanded version of DCL Memo 75, Department of Artificial Intelligence, U. of Edinburgh (1974). Also http://www.doc.ic.ac.uk/textasciitilderak/.

[Kowalski, 2011] R. Kowalski. Computational Logic and Human Thinking: How to be Artificially Intelligent, Cambridge University Press, 2011.

[Kowalski, 2013] R. Kowalski. Logic Programming in the 1970s. In: P. Cabalar and T.C. Son (eds.) LPNMR 2013. Springer Verlag, 2013.

[Kowalski and Kuehner, 1971] R. Kowalski and D. Kuehner. Linear Resolution with selection function, Artificial Intelligence, vol 2, 1971, 227-260, 1971.

[Kreisel, 1963] G. Kreisel. Generalized inductive definitions. Tech. rep., Stanford University, 1963.

[Kuehner, 1969] D. Kuehner. Bi-directional search with Horn clauses. Edinburgh University, 1969.

[Kunen, 1987] K. Kunen. Negation in logic programming. The Journal of Logic Programming, 4(4), 289-308, 1987.

[Lifschitz, 1988] V. Lifschitz. On the Declarative Semantics of Logic Programs with Negation, in: J. Minker (ed.), Foundations of Deductive Databases and Logic Programming, Morgan Kaufmann, Los Altos, CA, 1988, pp. 177-192.

[Lloyd, 1985/1987] J. W. Lloyd. Foundations of Logic Programming. New York: Springer Verlag (1985 and 1987)

[Lloyd and Torpor, 1985] J. W. Lloyd and R. W. Topor. A Basis for Deductive Database Systems. J. Logic Programming 2: 93-109, 1985.

[Loveland, 1968] D. W. Loveland. Mechanical theorem-proving by model elimination. Journal of the ACM, 15, 236-251, 1968. 
[Loveland, 1970] D. W. Loveland. A Linear Format for Resolution. In Symposium on Automatic Demonstra-tion, pp. 147-162. Springer, Berlin Heidelberg, 1970.

[Loveland, 1972] D. W. Loveland. A Unifying View of Some Linear Herbrand Procedures. JACM, 19(2), 366-384, 1972.

[Luckham, 1970] D. Luckham. Refinement Theorems in Resolution Theory. In Symposium on Automatic Demonstration (pp. 163-190). Springer, Berlin Heidelberg, 1970.

[Mariën et al., 2004] M. Mariën, D. Gilis, \& M. Denecker. On the relation between ID-logic and answer set programming. In Logics in Artificial Intelligence (pp. 108-120). Springer Berlin Heidelberg, 2004.

[Marek and Truszczyński, 1999] V. W. Marek and M. Truszczyński Stable models and an alternative logic programming paradigm. In The Logic Programming Paradigm (pp. 375-398). Springer Berlin Heidelberg, 1999.

[McCarthy and Hayes, 1969] J. McCarthy and P. Hayes. Some philosophical problems from the standpoint of artificial intelligence. In Meltzer, B. and Michie, D. and Swann, M. (eds.) Machine intelligence 4, Edinburgh University Press (pp. 463-502), 1969.

[McCarthy, 1980] J. McCarthy. Circumscription - a form of non-monotonic reasoning. Artificial intelligence, 13(1), 27-39, 1980.

[McDermott and Doyle, 1980] D. McDermott and J. Doyle. Non-monotonic logic I. Artificial intelligence,13(1), 41-72, 1980.

[Minker, 1996] J. Minker. Logic and databases: A 20 year retrospective (pp. 1-57). Springer Berlin Heidelberg, 1996.

[Minsky, 1975] M. Minsky. A framework for representing knowledge. In Winston, P. H. (ed.) The psychology of Computer Vistino. McGraw-Hill, New York, 211-277, 1975.

[Moore, 1985] R. C. Moore. Semantical considerations on nonmonotonic logic. Artificial intelligence, 25(1), 75-94, 1985

[Naqvi, 1988] S. A. Naqvi. A Logic for Negation in Database Systems, in: J. Minker (ed.), Foundations of Deductive Databases and Logic Programming, Morgan Kaufmann, Los Altos, CA, 1988, pp. 378-387.

[Nicolas and Gallaire, 1978] J. M. Nicolas and H. Gallaire. Database: Theory vs. Interpretation. In: Gallaire, H., Minker, J. (eds.), Logic and Databases, Plenum, New York, 1978.

[Niemelä, 1999] I. Niemelä. Logic programs with stable model semantics as a constraint programming paradigm. Annals of Mathematics and Artificial Intelligence, 25(3-4), 241-273, 1999.

[Nilsson, 1968] N. J. Nilsson. Searching problem-solving and game-playing trees for minimal cost solutions. In IFIP Congress (2) (pp. 1556-1562, 1968)

[Peirce, 1931] C. S. Peirce. Collected Papers. C. Hartshorn \& P. Weiss (eds.) Cambridge, MA: Harvard University Press, 1931.

[Pereira, 1984] L. M. Pereira. Logic Control with Logic, in: Implementations of Prolog, pp. 177-193, J. Campbell (ed.), Ellis Horwood, 1984

[Pereira et al., 1991] L. M. Pereira, J. N. Aparício, and J. J. Alferes. Hypothetical Reasoning with Well Founded Semantics. In SCAI (pp. 289-300), 1991.

[Pereira and Warren, 1980] F. C. Pereira and D. H. Warren. Definite clause grammars for language analysis - a survey of the formalism and a comparison with augmented transition networks. Artificial intelligence, 13(3), 231-278, 1980.

[Poole, 1988] D. Poole. A logical framework for default reasoning. Artificial intelligence, 36(1), $27-47,1988$.

[Poole et al., 1987] D. Poole, R. Goebel, and R. Aleliunas. Theorist: a logical reasoning system for defaults and diagnosis. In N. Cercone and G. McCalla (Eds.) The Knowledge Frontier: Essays in the Representation of Knowledge, Springer Verlag, New York, 331-352, 1987.

[Przymusinski, 1988] T. C. Przymusinski. On the Declarative Semantics of Deductive Databases and Logic Programs, In: J. Minker (ed.), Foundations of Deductive Databases and Logic Programming, Morgan Kaufmann, Los Altos, CA, 1988, pp. 193-216.

[Przymusinski, 1990] T. C. Przymusinski. The well-founded semantics coincides with the threevalued stable semantics. Fundamenta Informaticae, 13(4):445-463, 1990.

[Ramakrishnan and Ullman, 1995] R. Ramakrishnan and J. D. Ullman. A survey of deductive database systems. The journal of logic programming, 23(2), 125-149, 1995.

[Reiter, 1971] R. Reiter. Two Results on Ordering for Resolution with Merging and Linear Format. JACM 18(4), 630-646, 1971. 
[Reiter, 1978] R. Reiter. On Closed World Data Bases. In: Gallaire H. and Minker J. (eds.), Logic and Data Bases, Plenum Press, New York, pp. 55-76, 1978.

[Reiter, 1980] R. Reiter. A logic for default reasoning. Artificial intelligence, 13(1), 81-132, 1980.

Reiter, 1988] R. Reiter. On Integrity Constraints. In: 2nd Conference on Theoretical Aspects of Reasoning about Knowledge, 97-111, 1988.

[Robinson, 1965a] J. A. Robinson. A Machine-Oriented Logic Based on the Resolution Principle. JACM, 12(1) 23-41, 1965.

[Robinson, 1965b] J. A. Robinson. Automatic deduction with hyper-resolution, International J. Computer Math. 1, 3. 227-234, 1965.

[Sacca and Zaniolo, 1990] D. Sacca and C. Zaniolo. Stable models and non-determinism in logic programs with negation. In: Proceedings of the Ninth ACM SIGACT-SIGMOD-SIGART Symposium on Principles of Database Systems, ACM, 205-217, 1990.

[Sacca and Zaniolo, 1991] D. Sacca and C. Zaniolo. Partial Models and Three-Valued Models in Logic Programs with Negation. In LPNMR, 87-101, 1991.

[Sadri and Kowalski, 1988] F. Sadri and R. Kowalski. A Theorem-Proving Approach to Database Integrity. In: Minker, J. [ed.], Foundations of Deductive Databases and Logic Programming, Morgan Kaufmann, 313-362, 1988.

[Sagonas et al., 1994] K. Sagonas, T. Swift, and D. S. Warren. XSB as an Efficient Deductive Database Engine. Pro-ceedings of the ACM SIGMOD International Conference on the Management of Data pp. 442-453, 1994.

[Satoh and Iwayama, 1992] K. Satoh and N. Iwayama. Computing abduction using the TMS. Proceedings of ICLP. MIT Press 505-518, 1992.

[Scott, 1970] D. Scott. Outline of a mathematical theory of computation. Proc. of the Fourth Annual Princeton Conference on Information 5ciences and Systems, pp. 169-176, 1970.

[Shepherdson, 1988] J. Shepherdson. Negation in Logic Programming. In: Minker, J. [ed.], Foundations of Deductive Databases and Logic Programming, Morgan Kaufmann, 19-88, 1988.

[Smullyan, 1956] R. M. Smullyan. On definability by recursion (Abstract 782t). Bulletin AMS 62, 601, 1956.

[Smullyan, 1961] R. M. Smullyan. Theory of Formal Systems. Annals of Mathematical Studies Vol 47. Princeton University Press, Princeton, New Jersey, 1961.

[Tamaki and Sato, 1986] H. Tamaki and T. Sato. OLD Resolution with Tabulation. Third International Conference on Logic Programming pp. 84-98. Springer, Berlin, Heidelberg, 1986.

[Tekle and Liu, 2011] K. T. Tekle and Y. A. Liu. More Efficient Datalog Queries: Subsumptive Tabling beats Magic Sets. In Proceedings of SIGMOD International Conference on Management of Data 661-672, 2011.

[Thagard, 2005] P. Thagard. Mind: Introduction to Cognitive Science. Second edition. MIT Press, 2005.

[Ueda, 1986] K. Ueda. Guarded Horn Clauses: A Parallel Logic Programming Language with the Concept of a Guard. ICOT Technical Report TR-208, Institute for New Generation Computer Technology (ICOT), Tokyo, 1986. Revised version in Programming of Future Generation Computers, Nivat, M. and Fuchi, K. (eds.), North-Holland, Amsterdam, pp.441-456, 1988.

[Van Gelder, 1989] A. Van Gelder. Negation as failure using tight derivations for general logic programs, The Journal of Logic Programming, Volume 6, Issues 1-2, Pages 109-133, 1989.

[Van Gelder, 1993] A. Van Gelder. The alternating fixpoint of logic programs with negation, Journal of Computer and System Sciences, Volume 47, Issue 1, Pages 185-221, 1993.

[Van Gelder et al., 1991] A. Van Gelder, K. A. Ross, and J. Schlipf. The Well-Founded Semantics for General Logic Programs. JACM 38, 3, 620-650, 1991.

[Warren, 1978] D. H. Warren. Applied logic: its use and implementation as a programming tool. Ph.D. thesis. University of Edinburgh. Also Technical Note 290, AI Center, SRI International, 1978 .

[Warren, 1983] D. H. Warren). An abstract Prolog instruction set (Vol. 309). Menlo Park, California: SRI International, 1983.

[Warren et al., 1977] D. H. Warren, L. M. Pereira, and F. Pereira. Prolog - the language and its implementation compared with Lisp. In ACM SIGPLAN Notices (Vol. 12, No. 8, pp. 109-115). ACM. page 48. Warren et al.,. 1977.

[Winograd, 1971] T. Winograd. Procedures as a Representation for Data in a Computer Program for Under-standing Natural Language. MIT AI TR-235 (1971) Also: Understanding Natural Language. Academic Press, New York, 1972. 
[Wos et al., 1965] L. Wos, G. A . Robinson, and D. F. Carson. Efficiency and completeness of the set of support strategy in theorem proving. Journal of the ACM (JACM), 12(4), 536-541, 1965.

Wu et al., 2009] Y. Wu, M. Caminada, and D. M. Gabbay. Complete extensions in argumentation coincide with 3-valued stable models in logic programming. Studia logica, 93 (2-3), 383-403, 2009.

[Zamov and Sharonov, 1969] N. K. Zamov and V. I. Sharonov. On a Class of Strategies for the Resolution Method. Zapiski Nauchnykh Seminarov POMI, 16, 54-64, 1969. 